\title{
$o$-Quinones Derived from Tribenzotriquinacenes: Functionalization of Inner Bay Positions and Use for Single-Wing Extensions
}

Yu-Fei Zhang, ${ }^{\dagger}$ Wan-Fa Tian, ${ }^{\dagger}$ Xiao-Ping Cao, ${ }^{*}{ }^{\dagger}$ Dietmar Kuck, ${ }^{*,}$ and Hak-Fun $\mathrm{Chow}^{*}$,

${ }^{\dagger}$ State Key Laboratory of Applied Organic Chemistry and College of Chemistry and Chemical Engineering, Lanzhou University, Lanzhou, 730000, P. R. China ${ }^{\ddagger}$ Department of Chemistry and Center for Molecular Materials $\left(\mathrm{CM}_{2}\right)$, Bielefeld University, 33615 Bielefeld, Germany

${ }^{\S}$ Department of Chemistry, State Key Laboratory of Synthetic Chemistry and The Center of Novel Functional Molecules, The Chinese University of Hong Kong, Shatin, Hong Kong E-mail: caoxplzu@163.com,dietmar.kuck@uni-bielefeld.de,hfchow@cuhk.edu.hk

\section{Table of Contents}

(1) ${ }^{1} \mathrm{H},{ }^{13} \mathrm{C}$ NMR and DEPT 135 spectra of all new compounds $\mathbf{6 a}, \mathbf{7 a}, \mathbf{6 b}, \mathbf{7 b}$,

11, 12, 14-25, 27a-f, 30, and 32; the HMBC spectrum of 24.

S2-S28

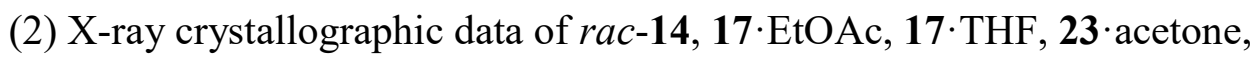

$18,27 \mathbf{b}, 27 \mathbf{c}$, and 32 (endo-anti-stereoisomer).

S29-S32

(3) Crystal packing of catechols $\mathbf{1 7}$ and $\mathbf{2 3}$ in different solvents.

(4) Crystal packing of phenazines $27 \mathbf{b}$ and $\mathbf{2 7 c}$. 
(1) ${ }^{1} \mathrm{H},{ }^{13} \mathrm{C}$ NMR and DEPT 135 spectra of all new compounds.

${ }^{1} \mathrm{H}\left(400 \mathrm{MHz}, \mathrm{CDCl}_{3}\right)$ and ${ }^{13} \mathrm{C}$ NMR $\left(100 \mathrm{MHz}, \mathrm{CDCl}_{3}\right)$ spectra of $\mathbf{6 a}$

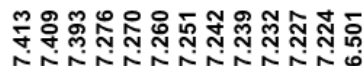

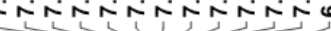



$\dot{i}$
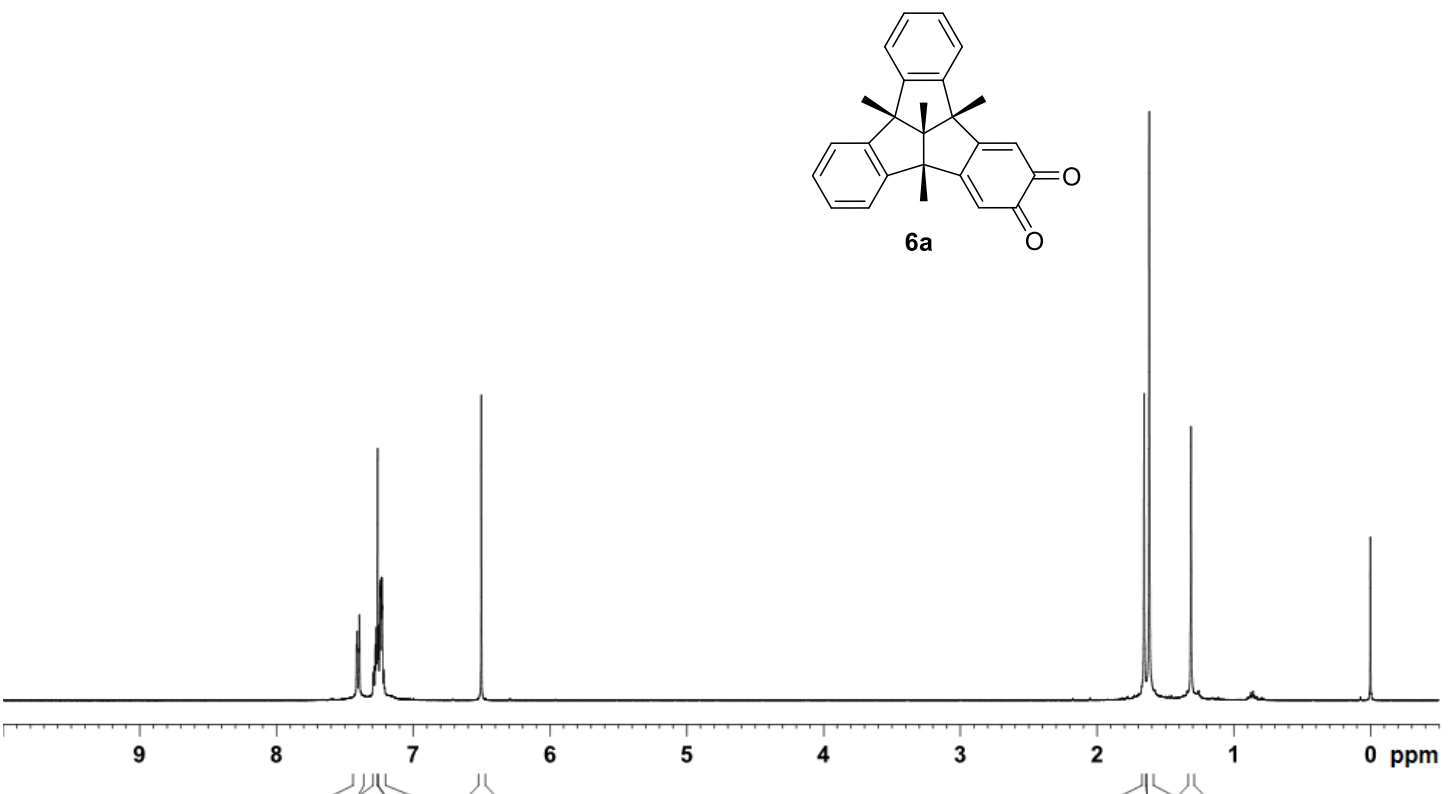
商|

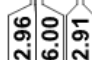

|
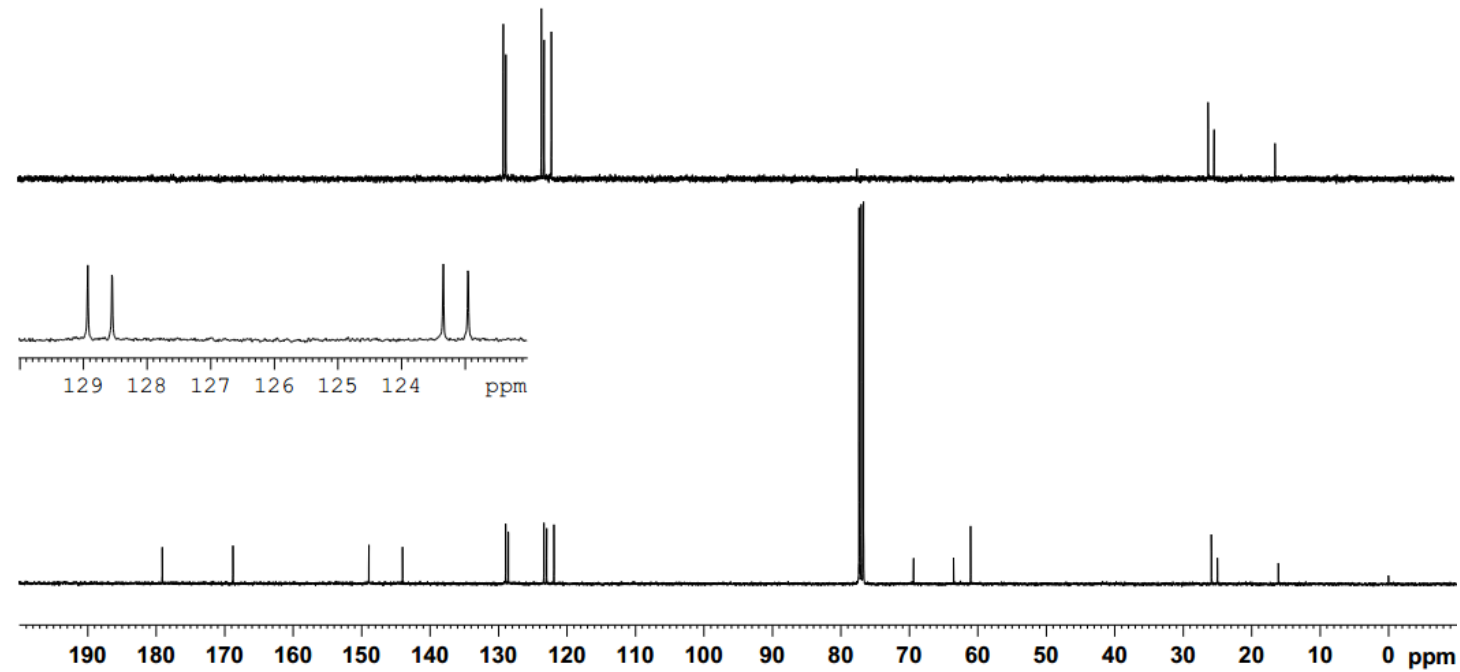
${ }^{1} \mathrm{H}\left(400 \mathrm{MHz}, \mathrm{CDCl}_{3}\right)$ and ${ }^{13} \mathrm{C} \mathrm{NMR}\left(100 \mathrm{MHz}, \mathrm{CDCl}_{3}\right)$ spectra of 7a

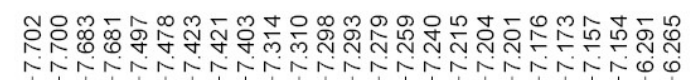

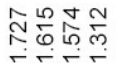
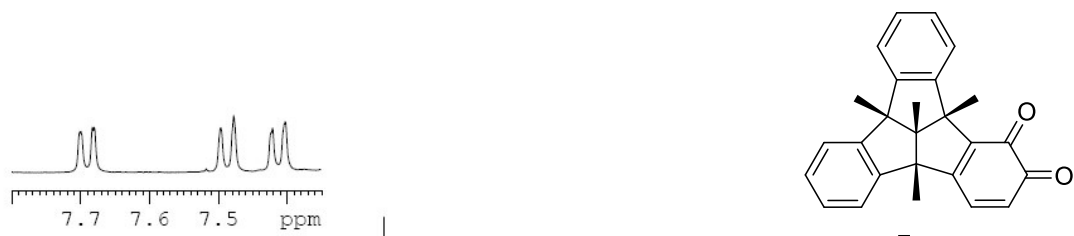

$7 a$

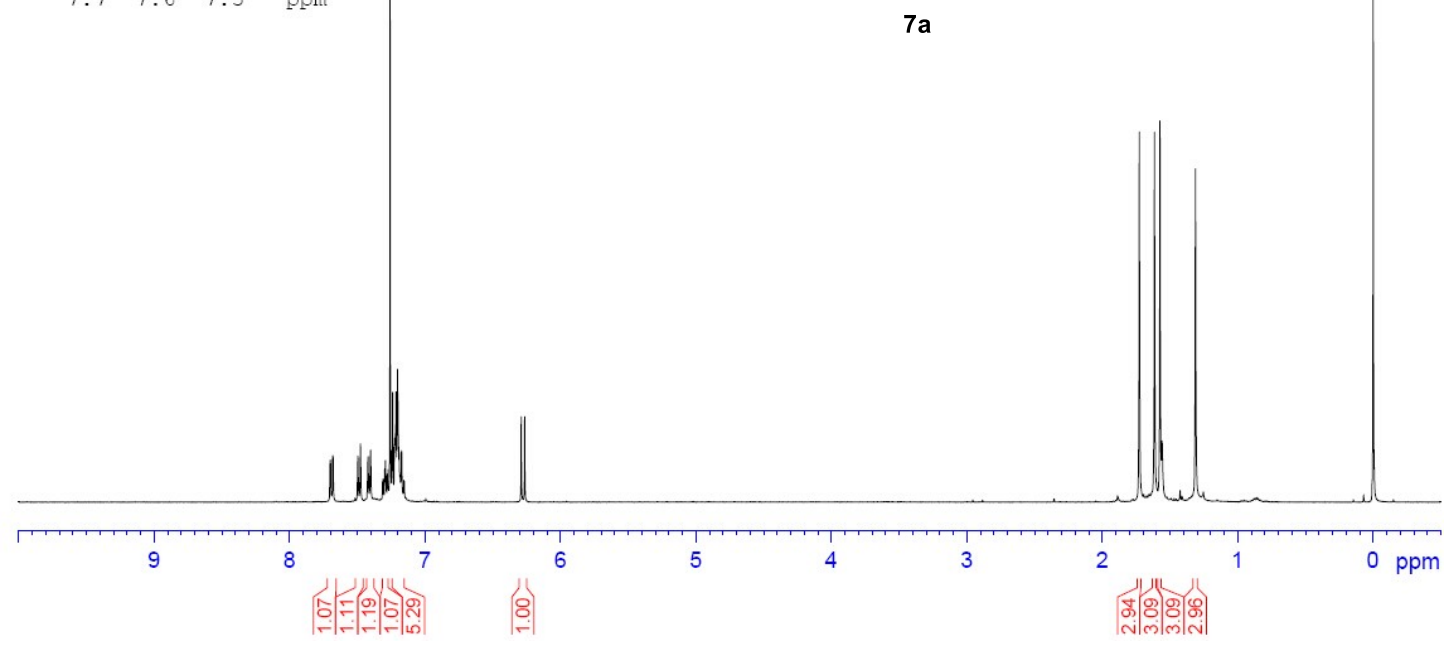

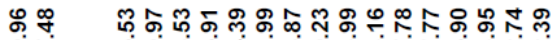

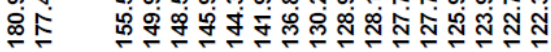

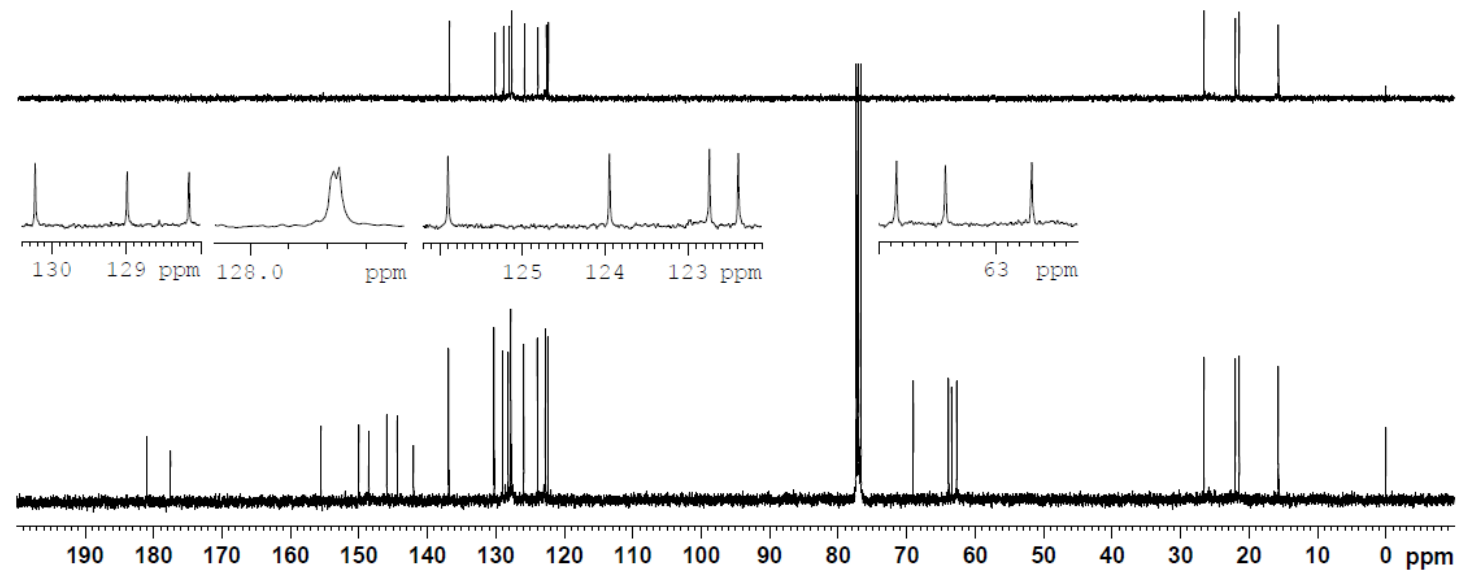


${ }^{1} \mathrm{H}\left(400 \mathrm{MHz}, \mathrm{CDCl}_{3}\right)$ and ${ }^{13} \mathrm{C} \mathrm{NMR}\left(100 \mathrm{MHz}, \mathrm{CDCl}_{3}\right)$ spectra of $\mathbf{6 b}$

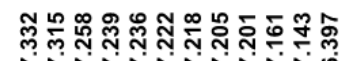

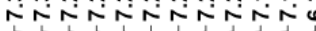

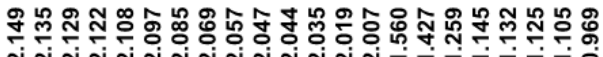

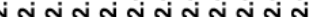
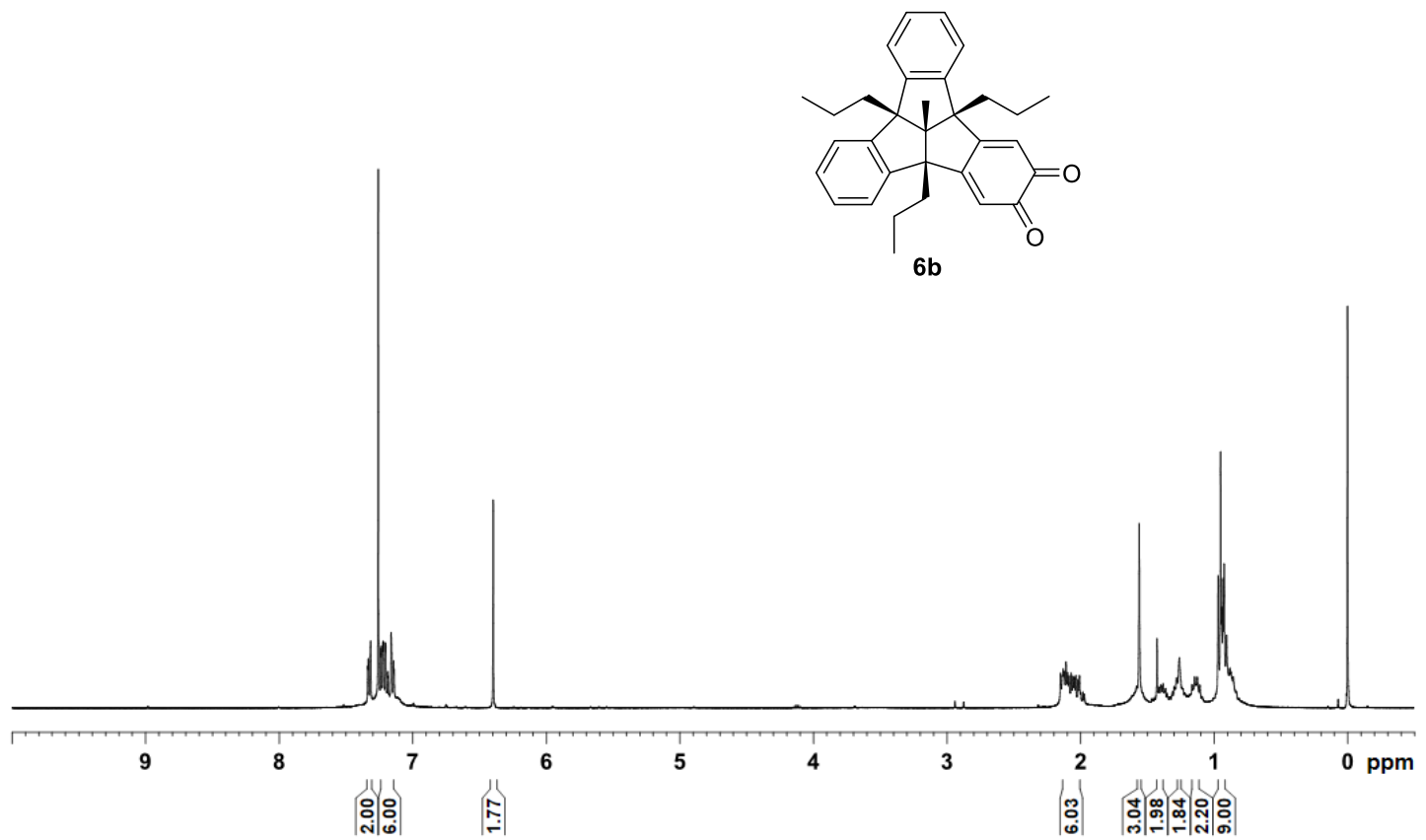

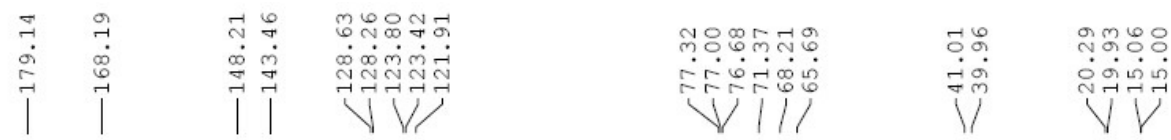

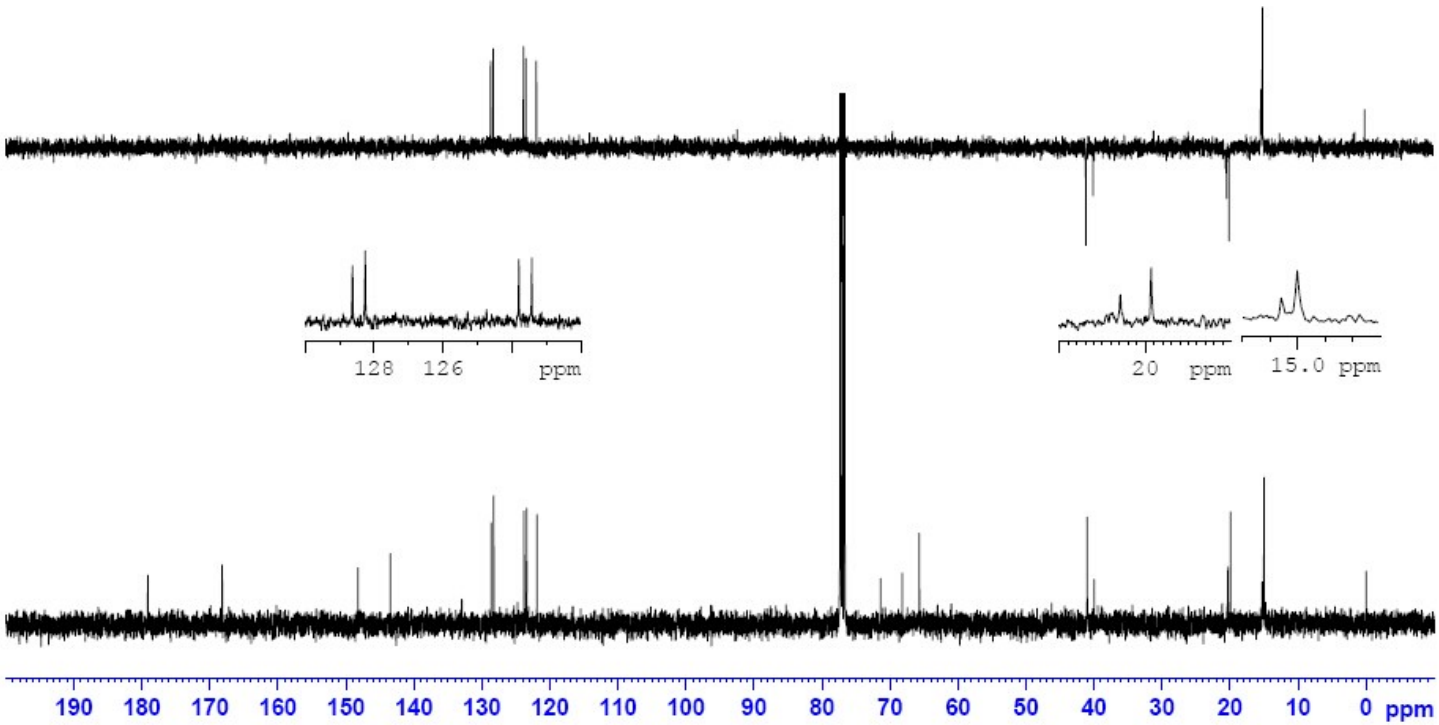


${ }^{1} \mathrm{H}\left(400 \mathrm{MHz}, \mathrm{CDCl}_{3}\right)$ and ${ }^{13} \mathrm{C} \mathrm{NMR}\left(100 \mathrm{MHz}, \mathrm{CDCl}_{3}\right)$ spectra of $7 \mathbf{b}$

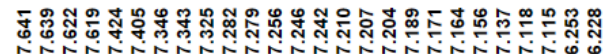

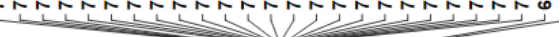
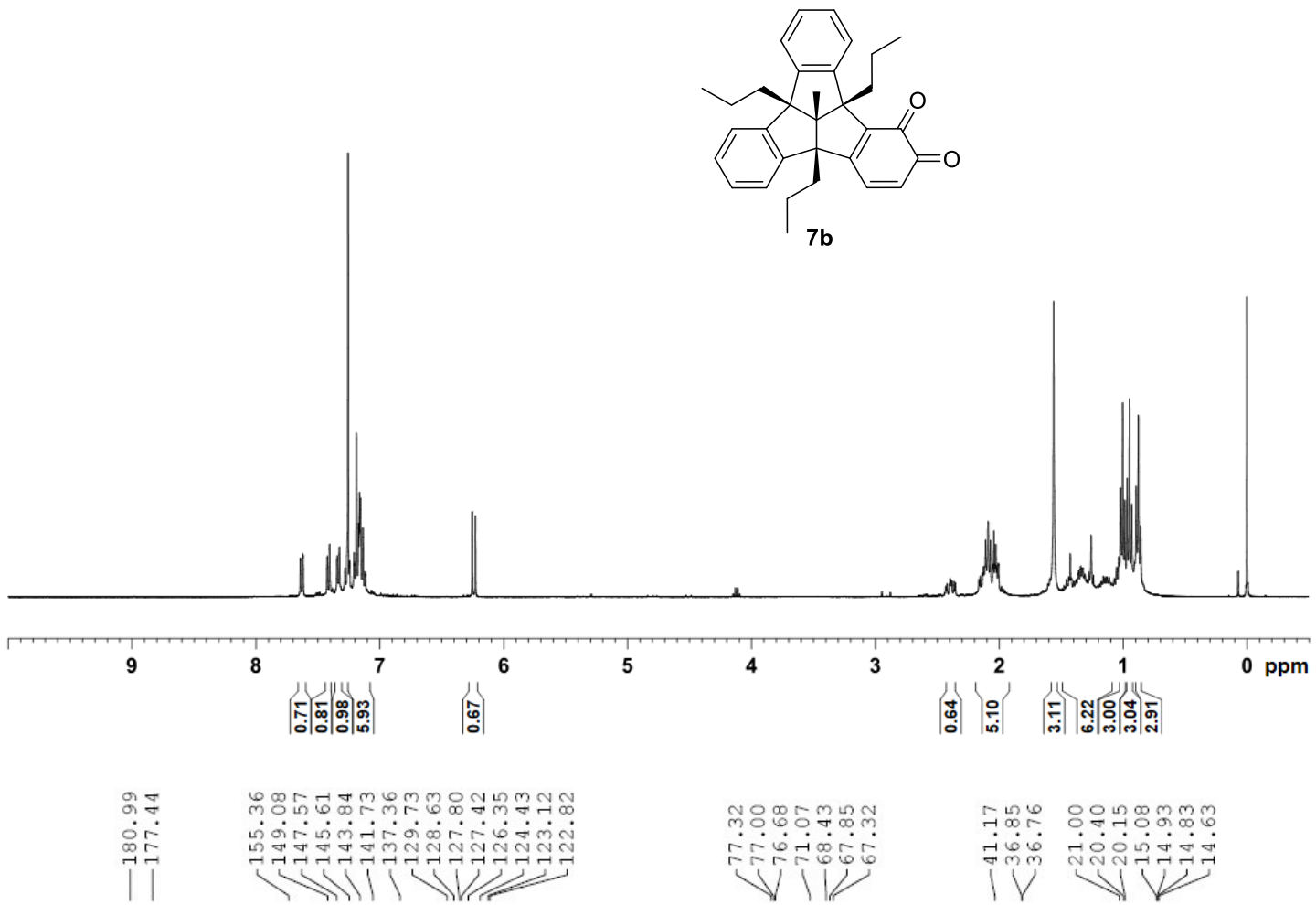

$7 b$
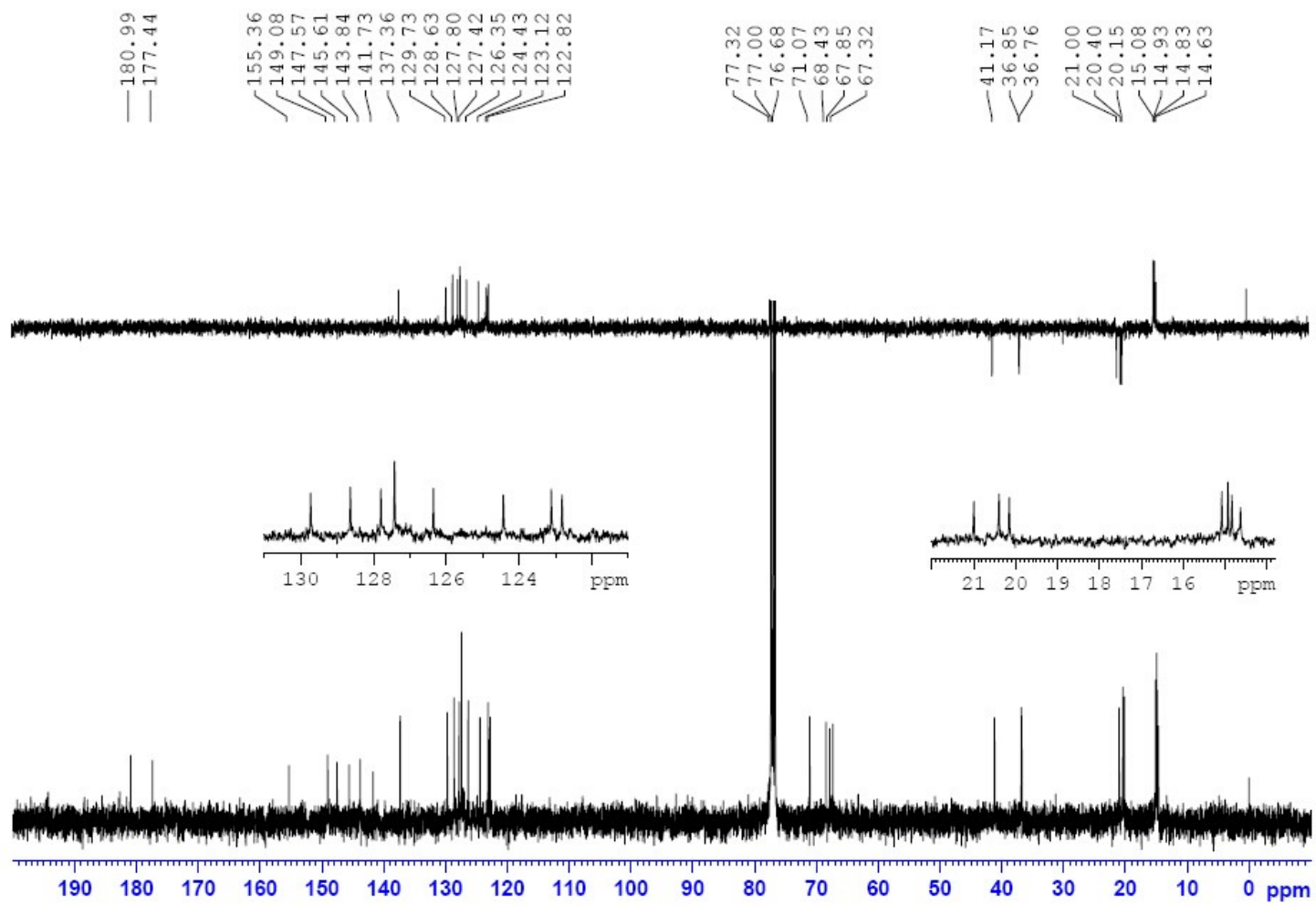
${ }^{1} \mathrm{H}\left(400 \mathrm{MHz}, \mathrm{CDCl}_{3}\right)$ and ${ }^{13} \mathrm{C} \mathrm{NMR}\left(100 \mathrm{MHz}, \mathrm{CDCl}_{3}\right)$ spectra of 11

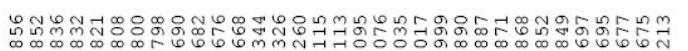

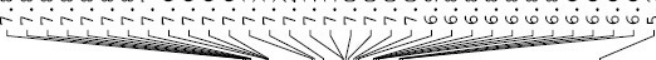
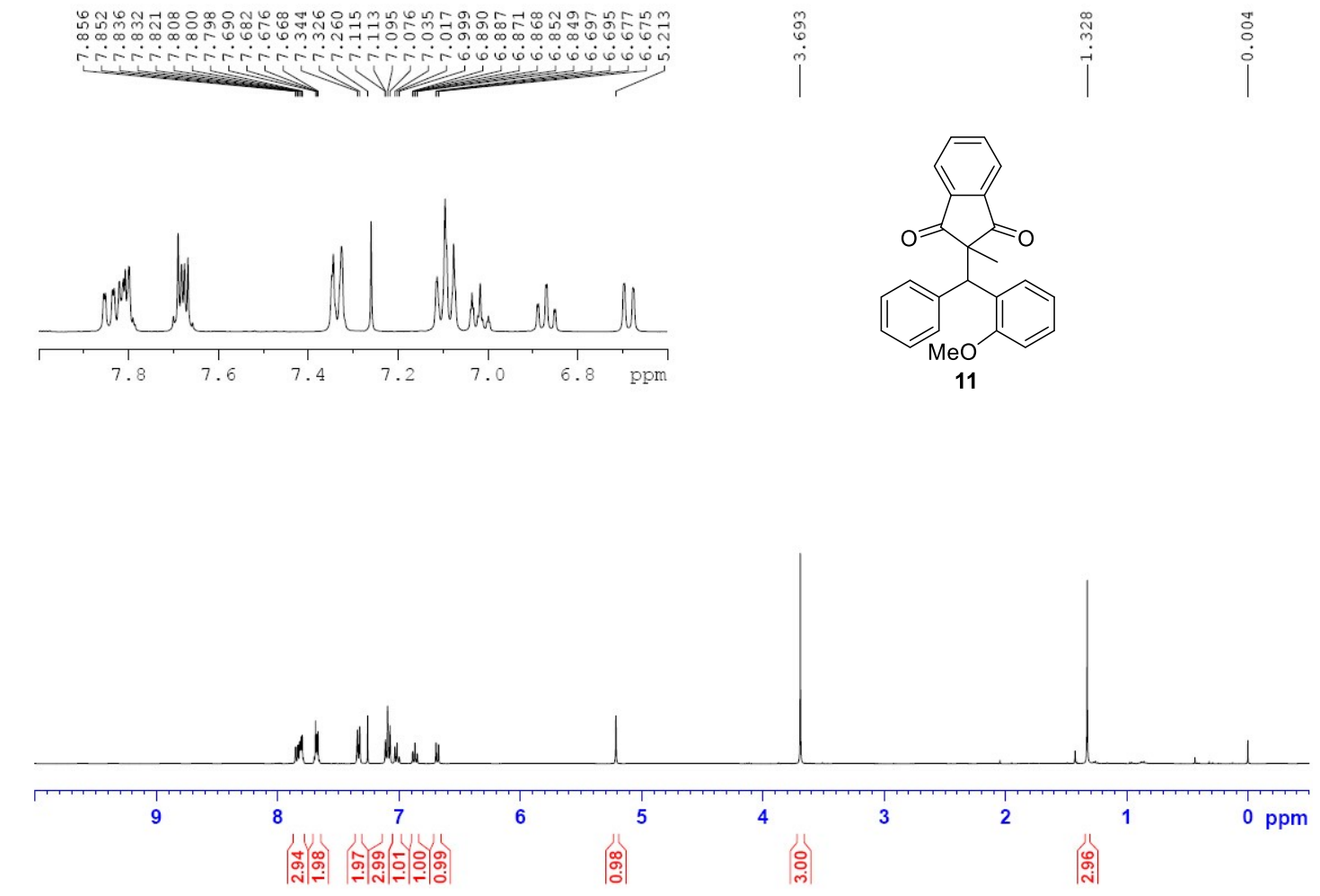

लू

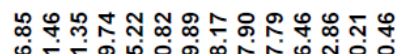

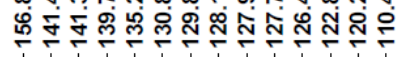
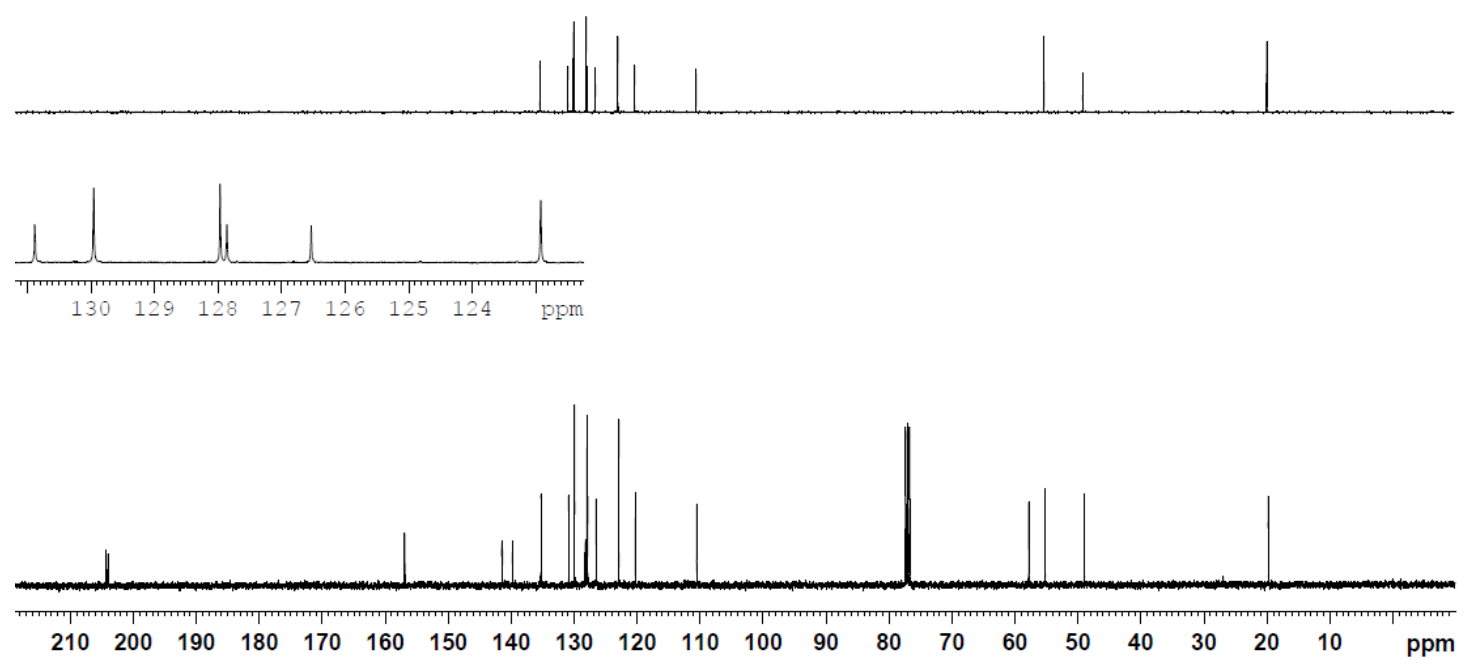
${ }^{1} \mathrm{H}\left(400 \mathrm{MHz}, \mathrm{CDCl}_{3}\right)$ and ${ }^{13} \mathrm{C} \mathrm{NMR}\left(100 \mathrm{MHz}, \mathrm{CDCl}_{3}\right)$ spectra of 12

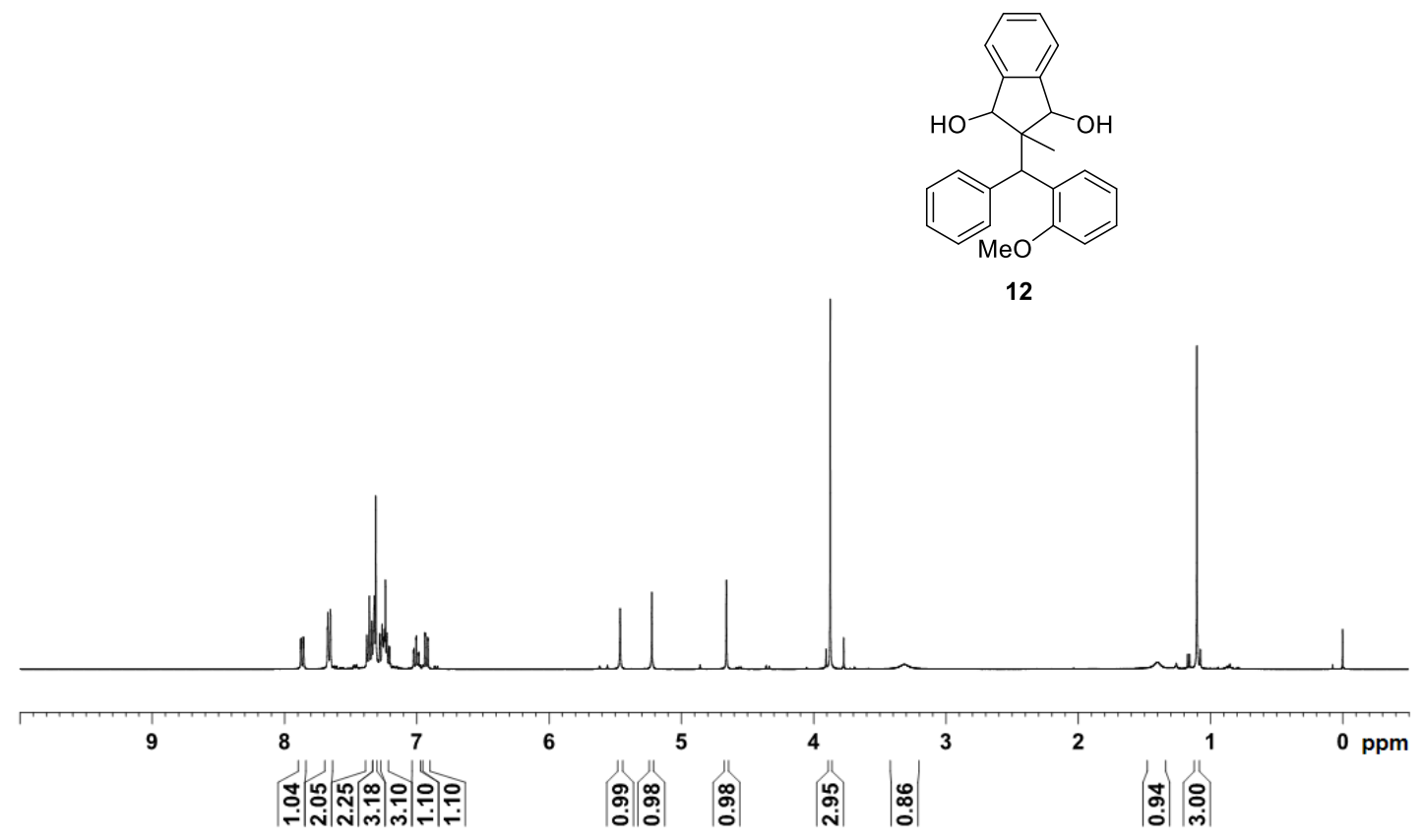

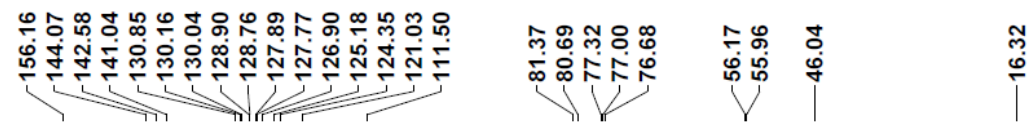
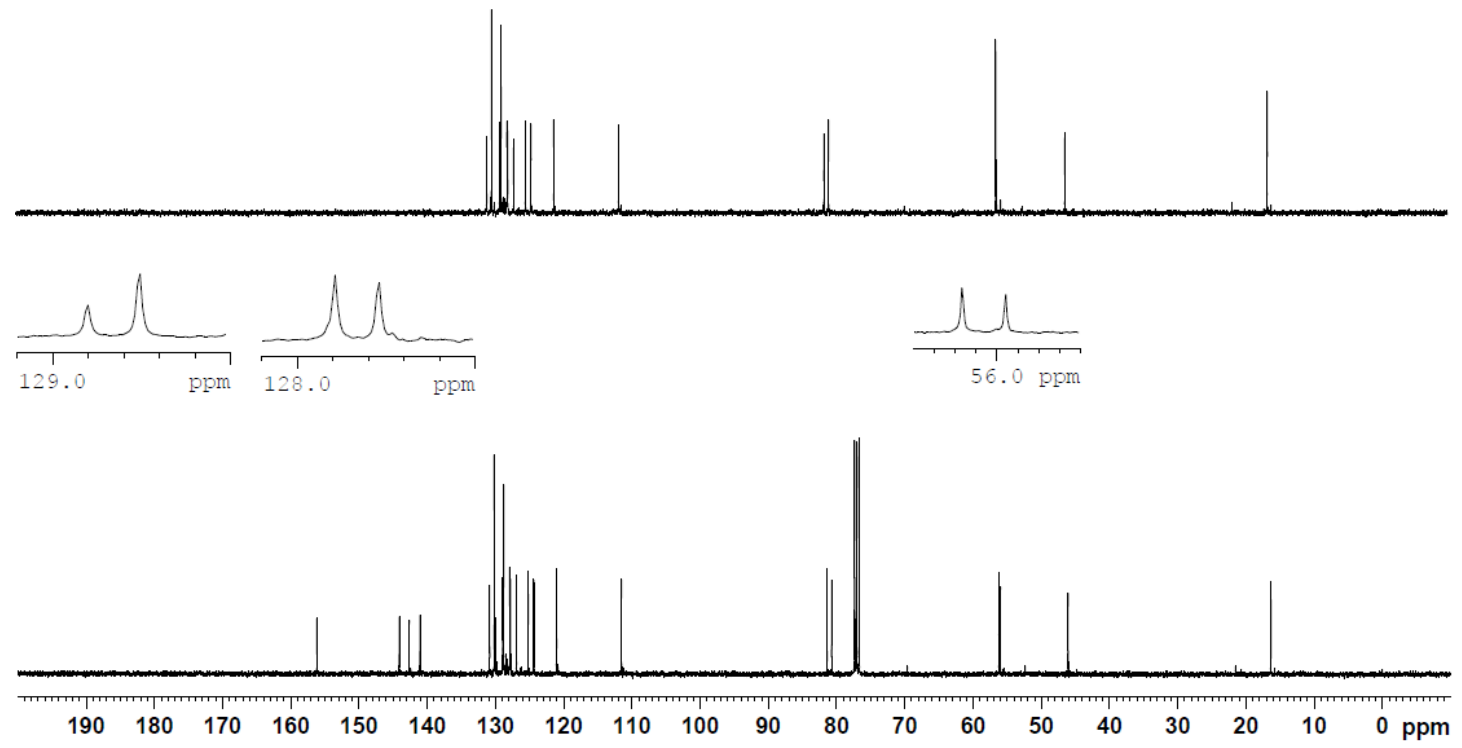
${ }^{1} \mathrm{H}\left(400 \mathrm{MHz}, \mathrm{CDCl}_{3}\right)$ and ${ }^{13} \mathrm{C} \mathrm{NMR}\left(100 \mathrm{MHz}, \mathrm{CDCl}_{3}\right)$ spectra of 14

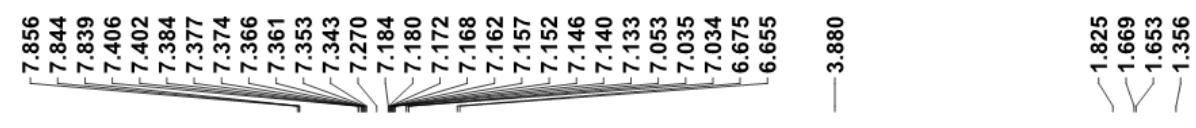

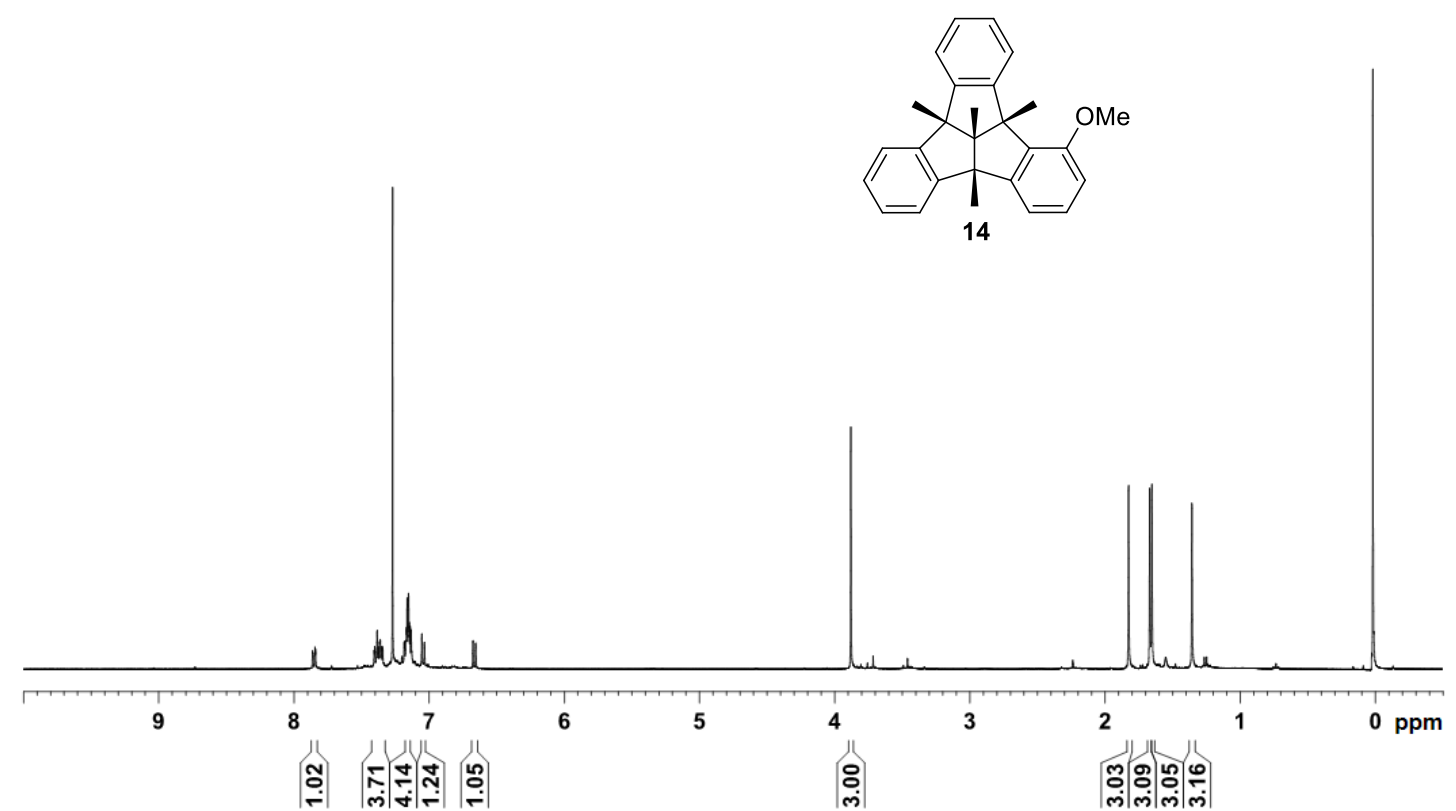

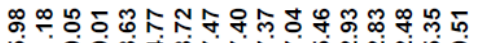

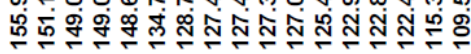

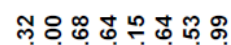

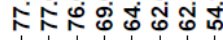

จุํํำ응

บ

io

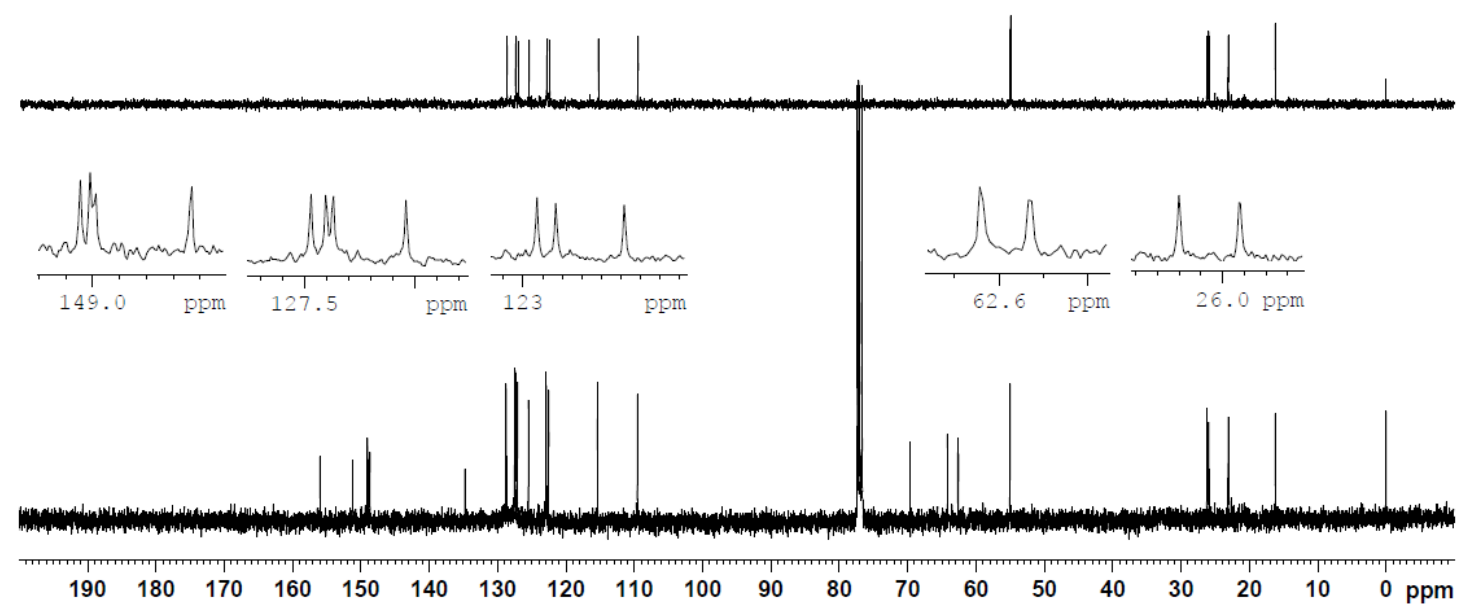


${ }^{1} \mathrm{H}\left(600 \mathrm{MHz}, \mathrm{CDCl}_{3}\right)$ and ${ }^{13} \mathrm{C} \mathrm{NMR}\left(150 \mathrm{MHz}, \mathrm{CDCl}_{3}\right)$ spectra of 15
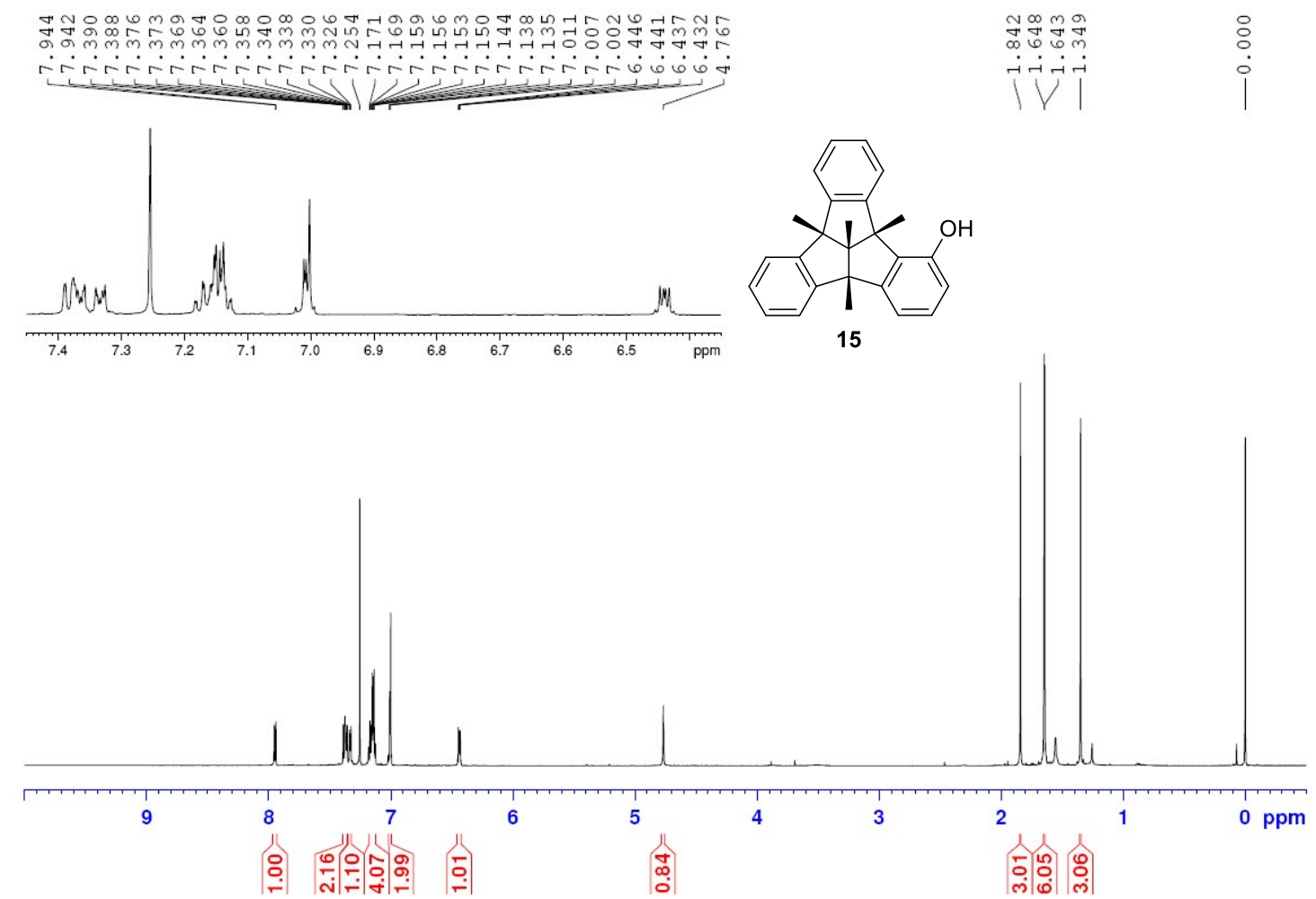

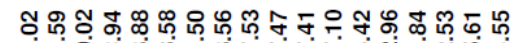

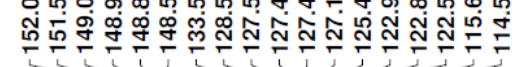

トКํำ

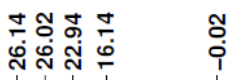

Nij|

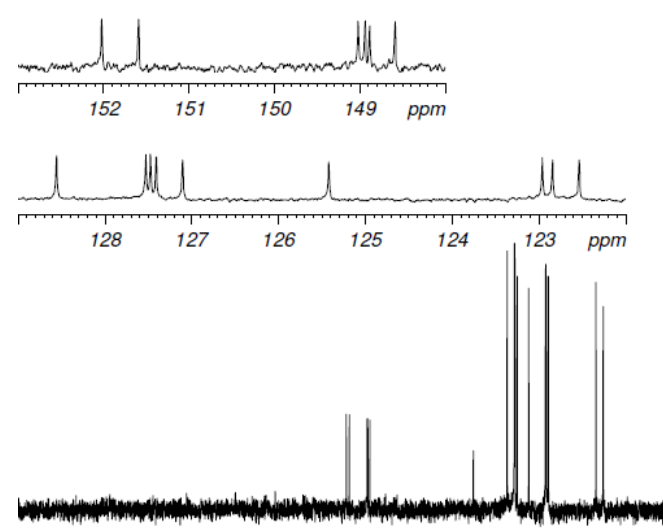

$\begin{array}{llllllll}190 & 180 & 170 & 160 & 150 & 140 & 130 & 120\end{array}$

$90 \quad 80$

70

$50 \quad 40 \quad 30$

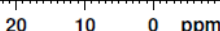


${ }^{1} \mathrm{H}\left(400 \mathrm{MHz}, \mathrm{CDCl}_{3}\right)$ and ${ }^{13} \mathrm{C} \mathrm{NMR}\left(100 \mathrm{MHz}, \mathrm{CDCl}_{3}\right)$ spectra of 16

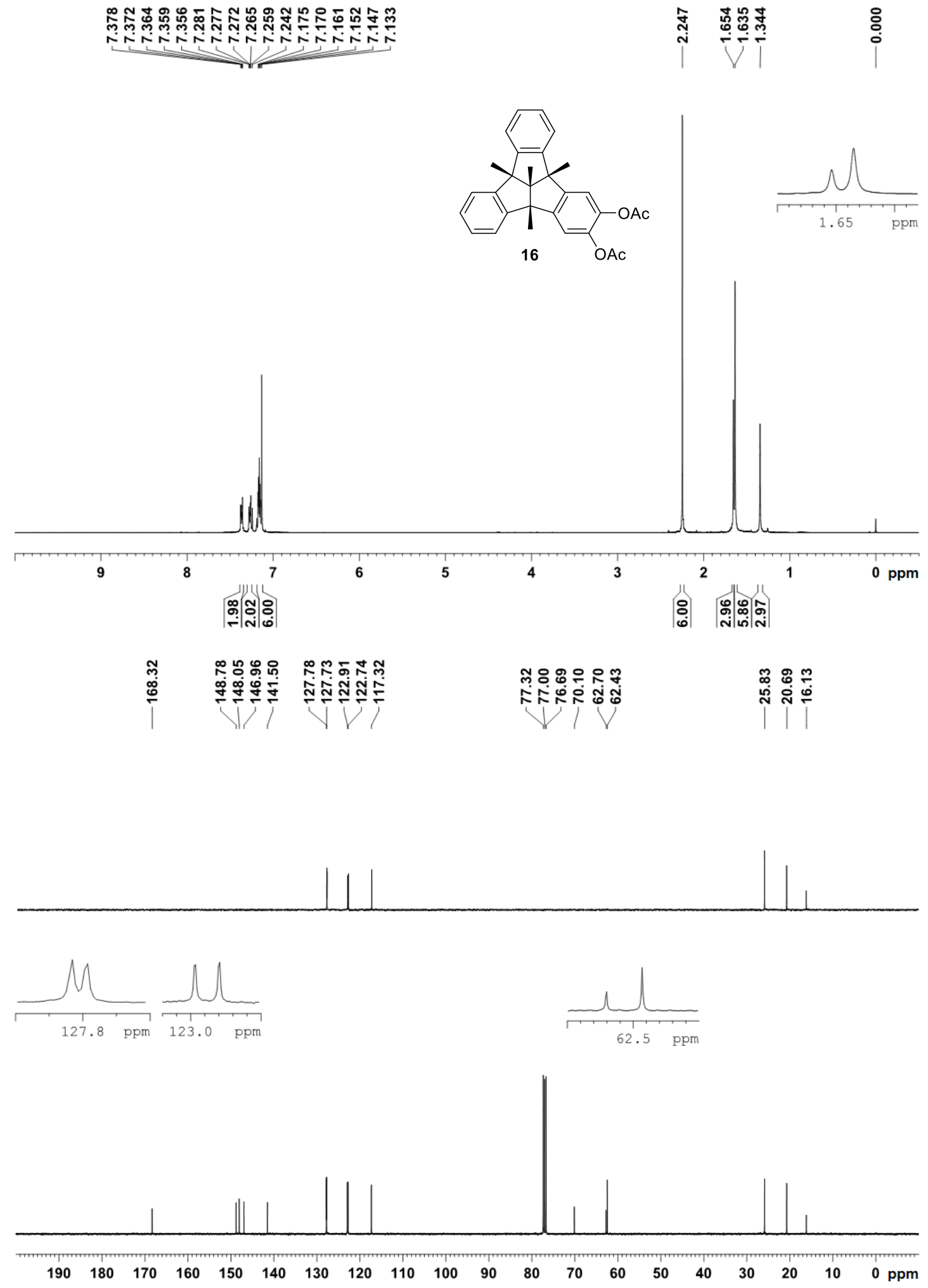


${ }^{1} \mathrm{H}\left(400 \mathrm{MHz}, \mathrm{CDCl}_{3}\right)$ and ${ }^{13} \mathrm{C} \mathrm{NMR}\left(100 \mathrm{MHz}, \mathrm{CDCl}_{3}\right)$ spectra of 17
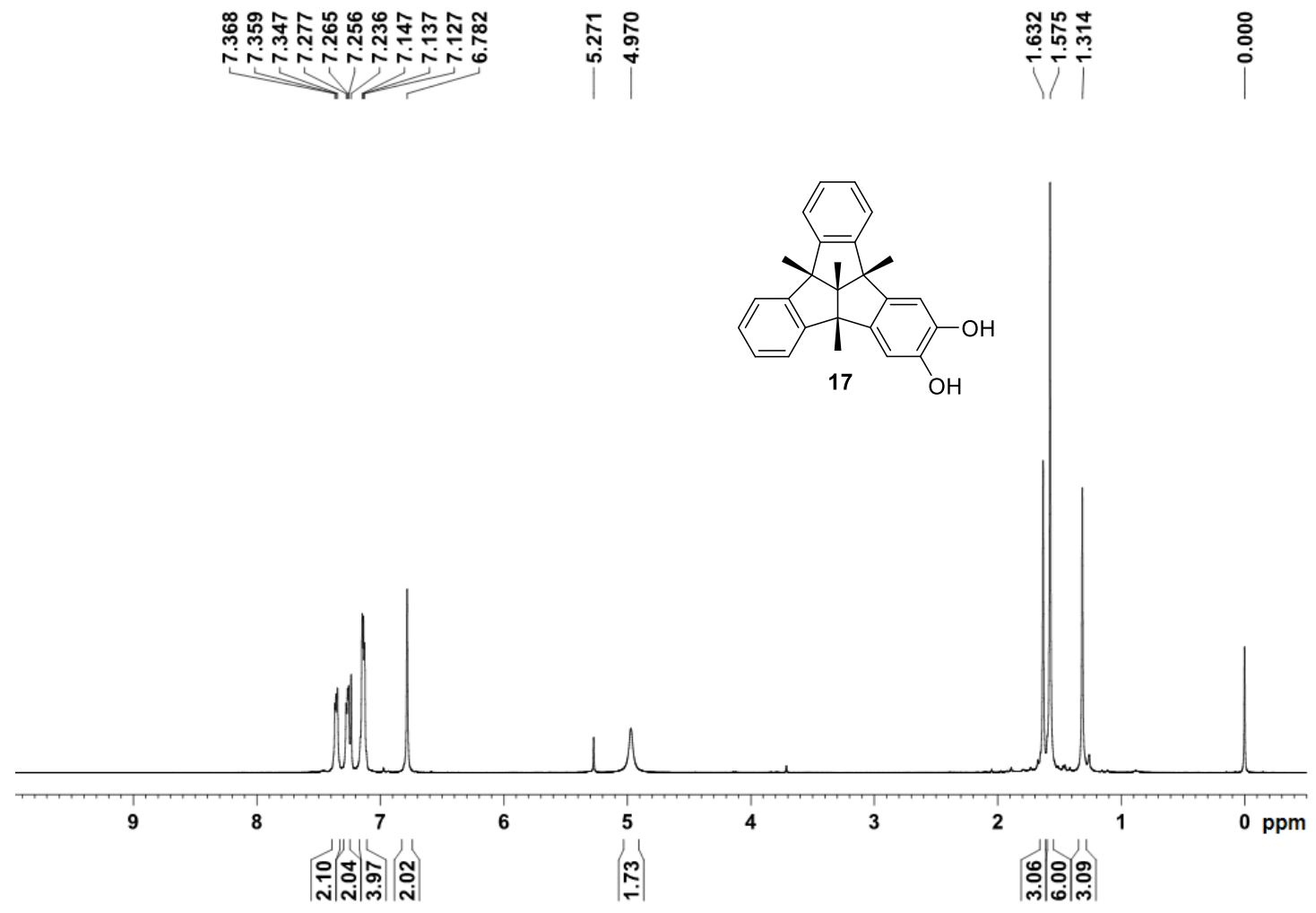

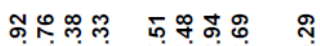

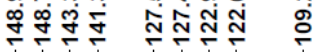

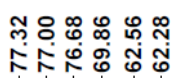

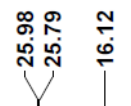

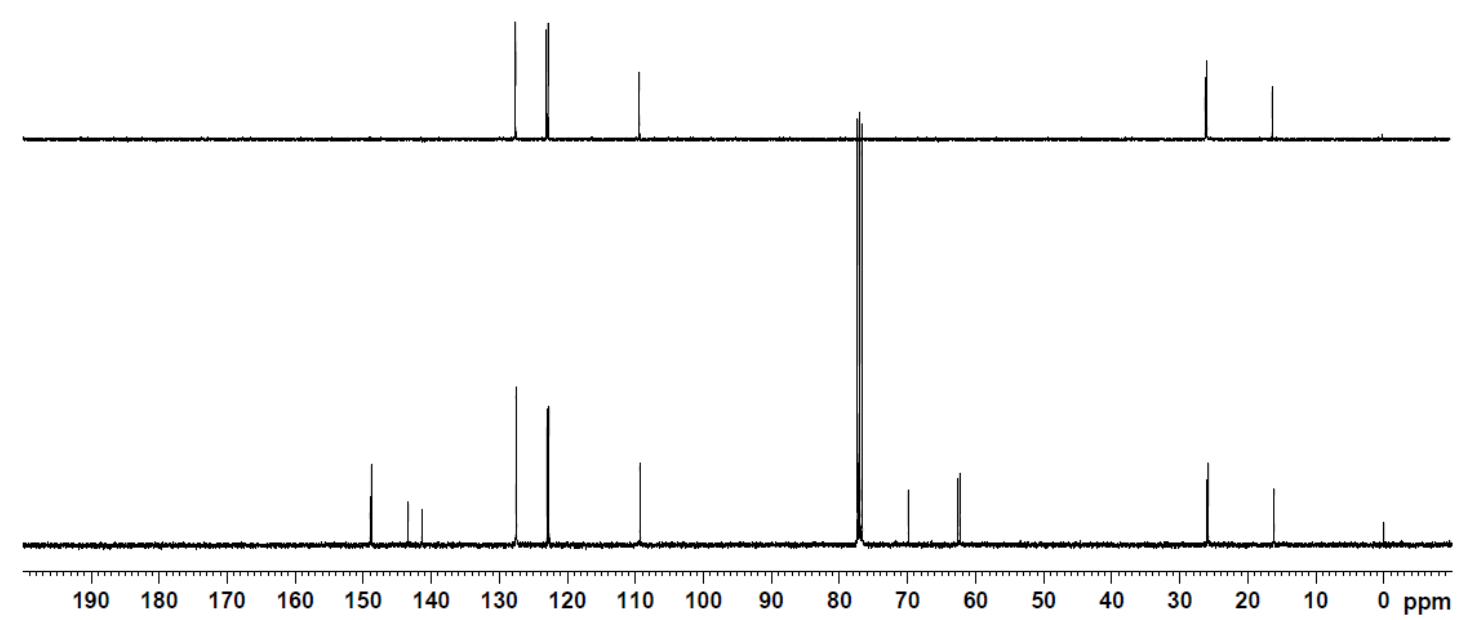


${ }^{1} \mathrm{H}\left(400 \mathrm{MHz}, \mathrm{CDCl}_{3}\right)$ and ${ }^{13} \mathrm{C} \mathrm{NMR}\left(100 \mathrm{MHz}, \mathrm{CDCl}_{3}\right)$ spectra of 18

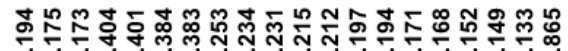

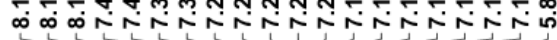

i

$\stackrel{\circ}{\circ}$
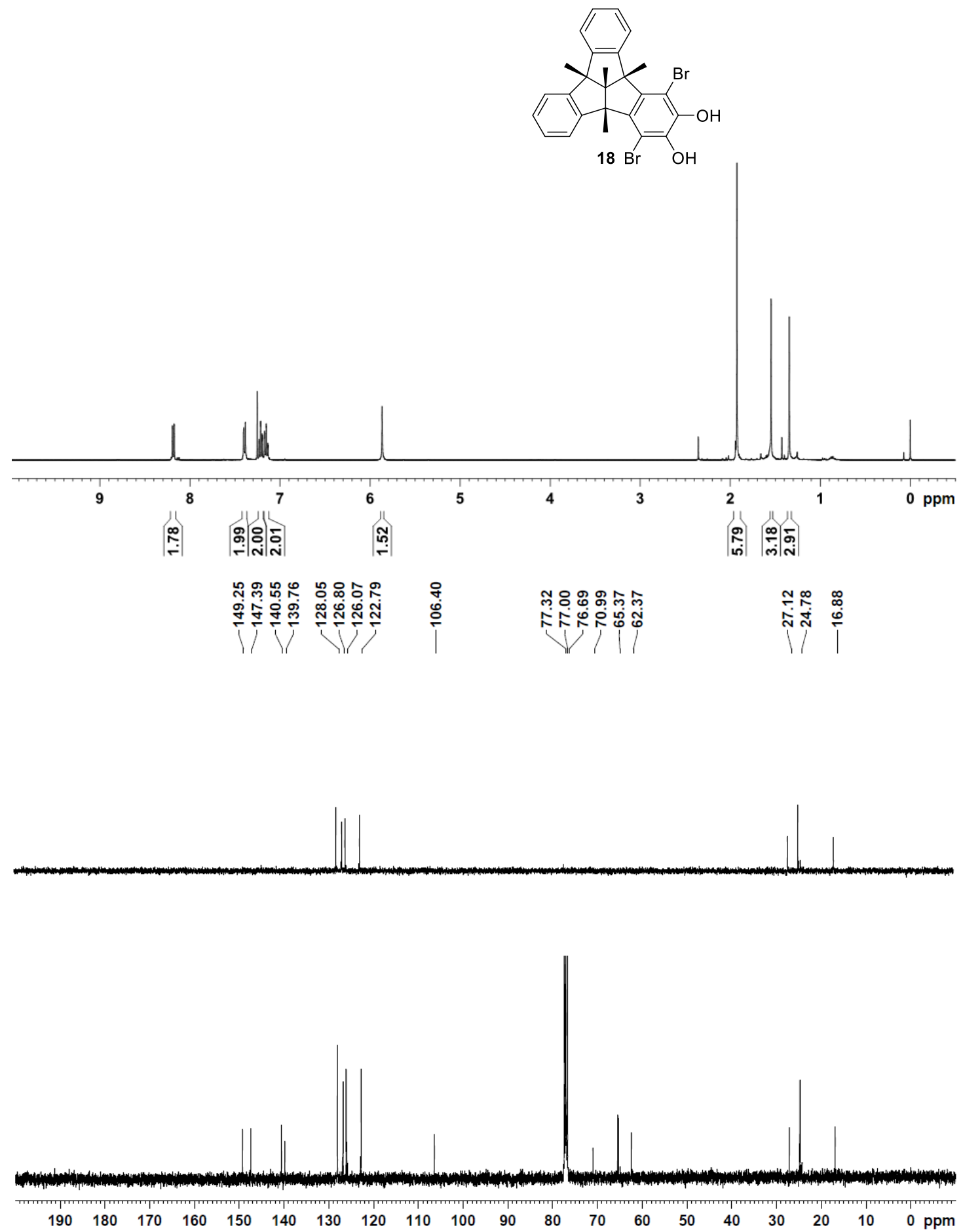
${ }^{1} \mathrm{H}\left(400 \mathrm{MHz}, \mathrm{CDCl}_{3}\right)$ and ${ }^{13} \mathrm{C} \mathrm{NMR}\left(100 \mathrm{MHz}, \mathrm{CDCl}_{3}\right)$ spectra of 19

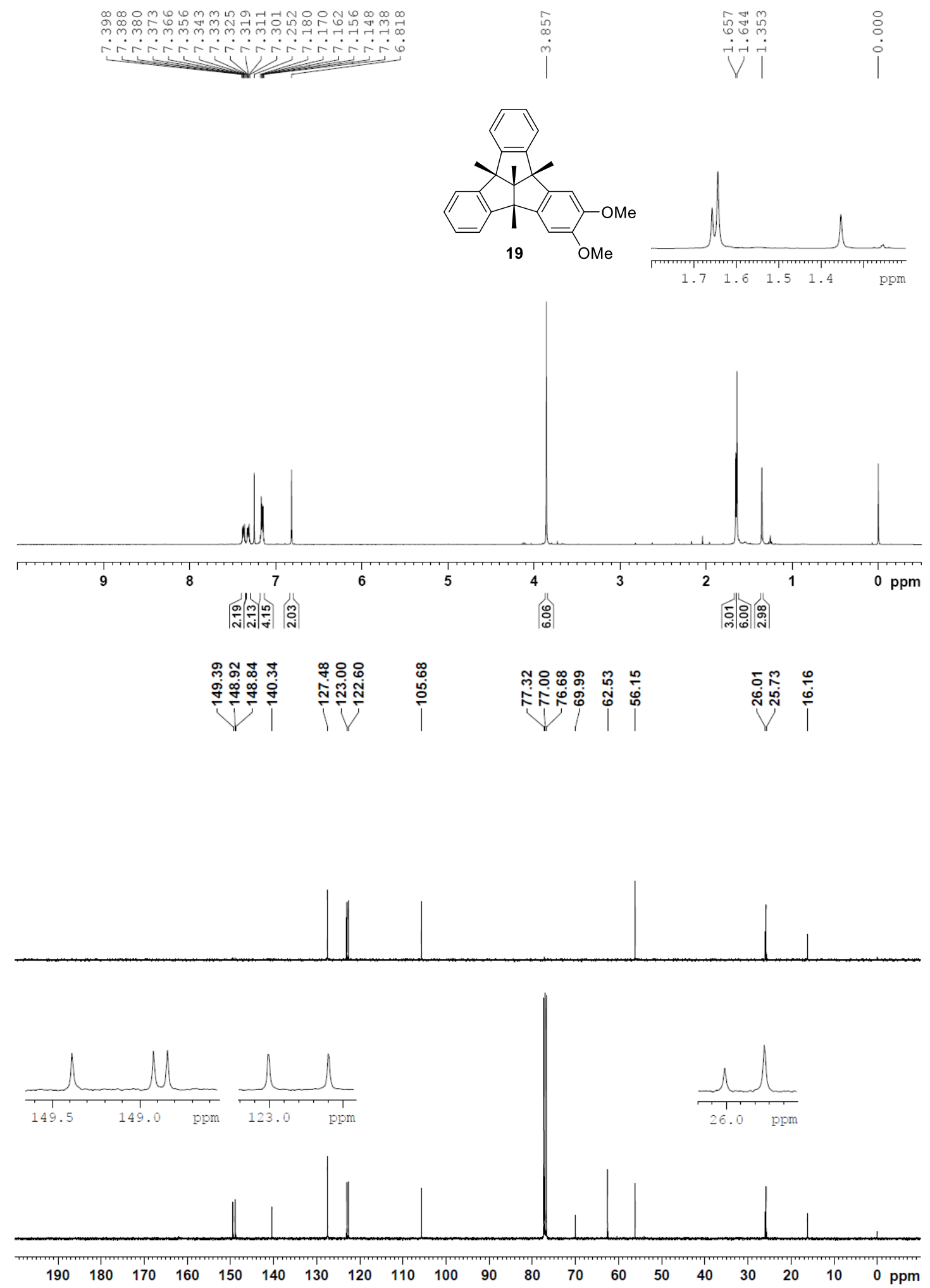


${ }^{1} \mathrm{H}\left(400 \mathrm{MHz}, \mathrm{CDCl}_{3}\right)$ and ${ }^{13} \mathrm{C}$ NMR $\left(100 \mathrm{MHz}, \mathrm{CDCl}_{3}\right)$ spectra of 20

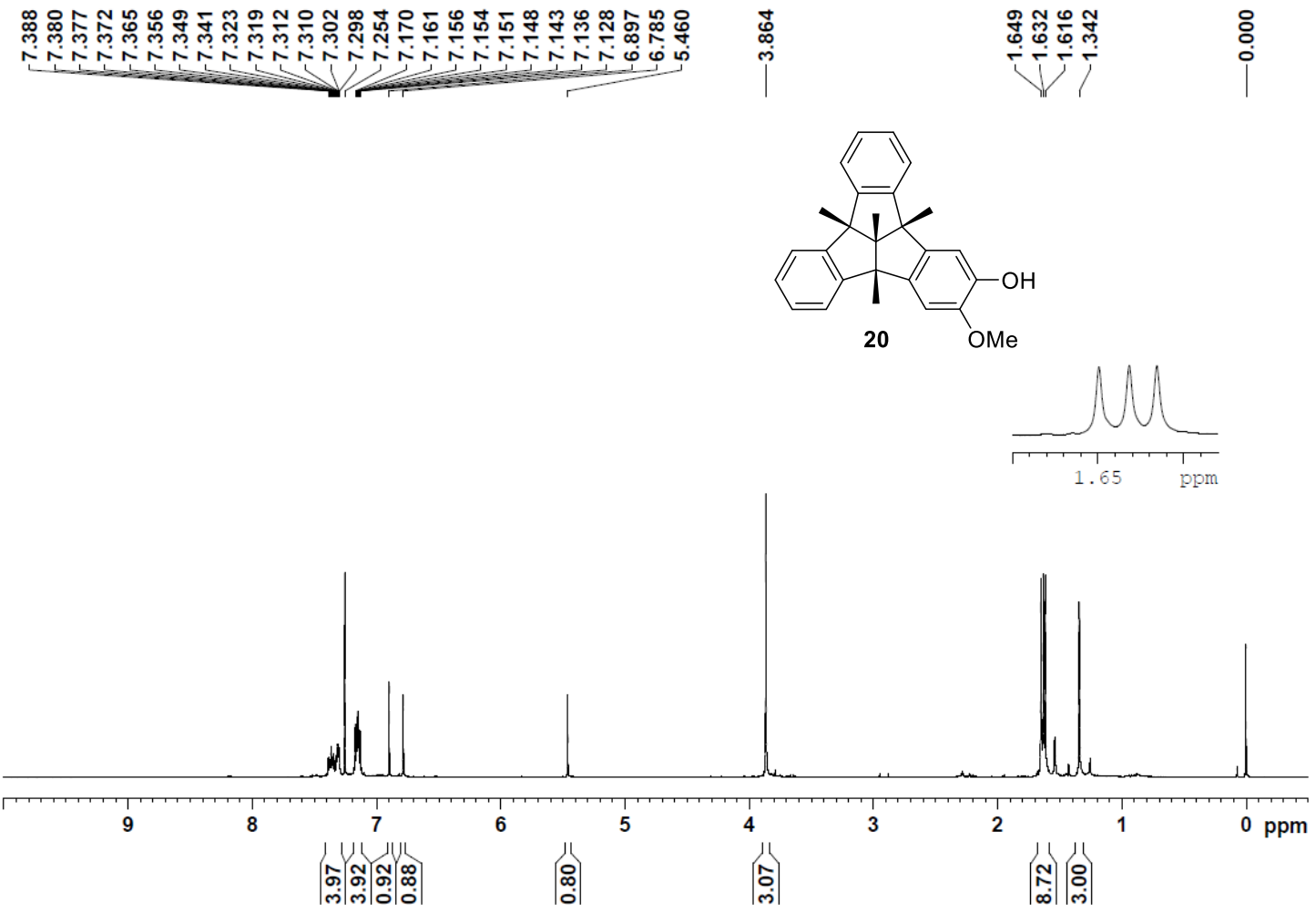

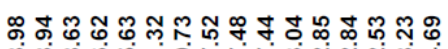

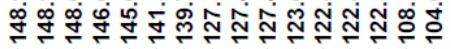

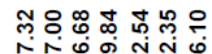

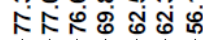

용ํำ ลู่ ล่ Y V
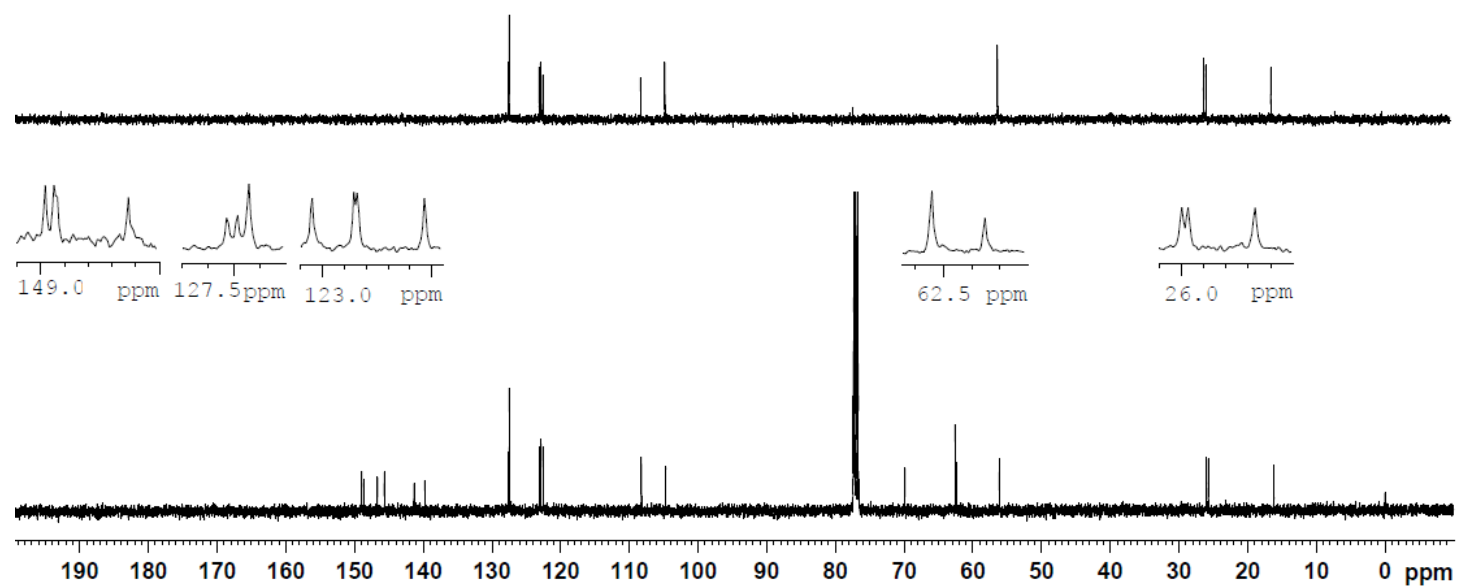
${ }^{1} \mathrm{H}\left(400 \mathrm{MHz}, \mathrm{CDCl}_{3}\right)$ and ${ }^{13} \mathrm{C} \mathrm{NMR}\left(100 \mathrm{MHz}, \mathrm{CDCl}_{3}\right)$ spectra of 21
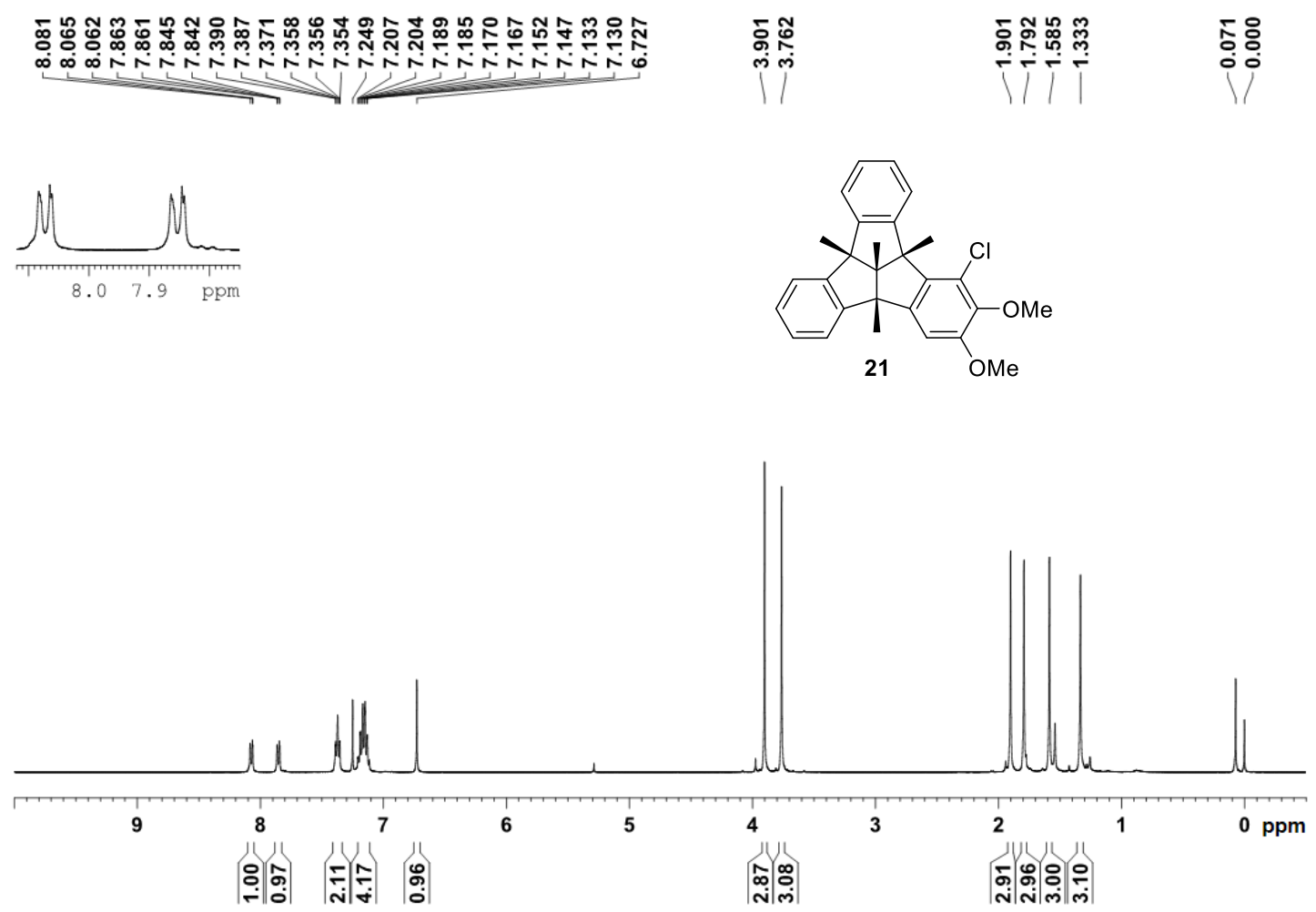

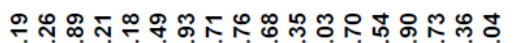

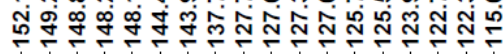
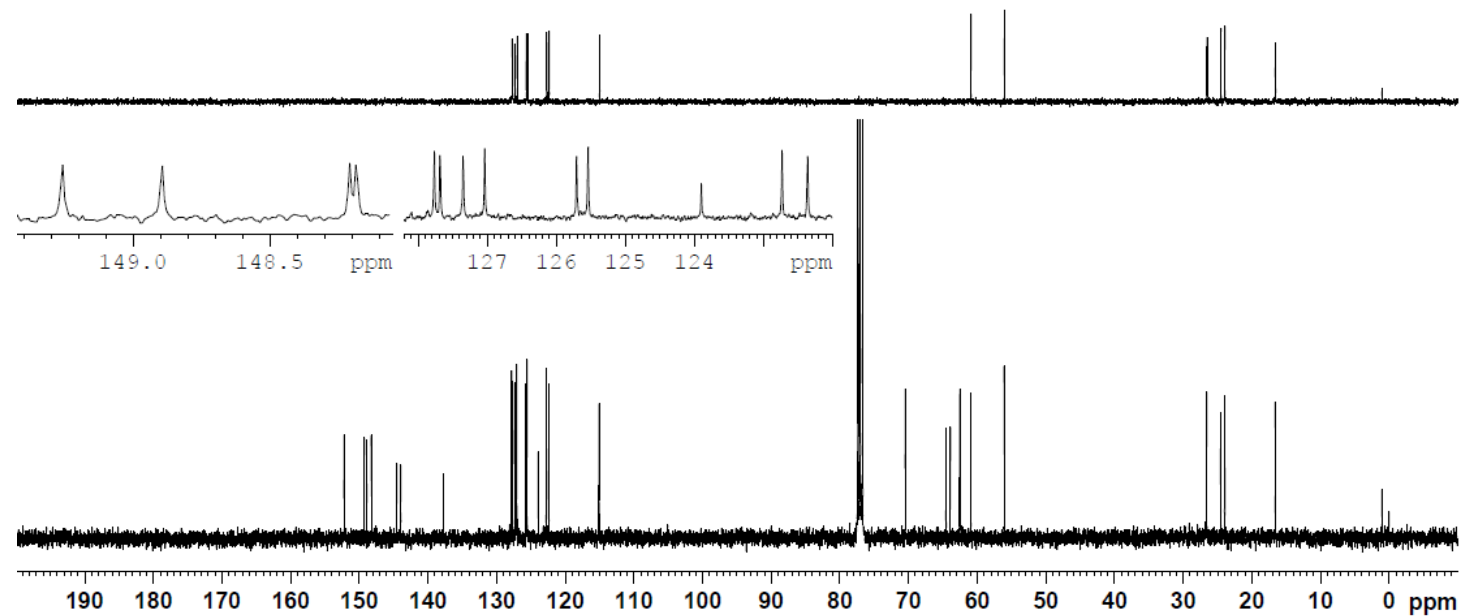
${ }^{1} \mathrm{H}\left(400 \mathrm{MHz}, \mathrm{CDCl}_{3}\right)$ and ${ }^{13} \mathrm{C} \mathrm{NMR}\left(100 \mathrm{MHz}, \mathrm{CDCl}_{3}\right)$ spectra of 22

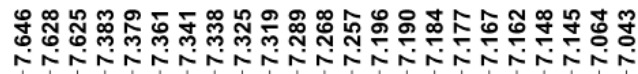

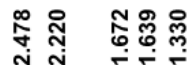
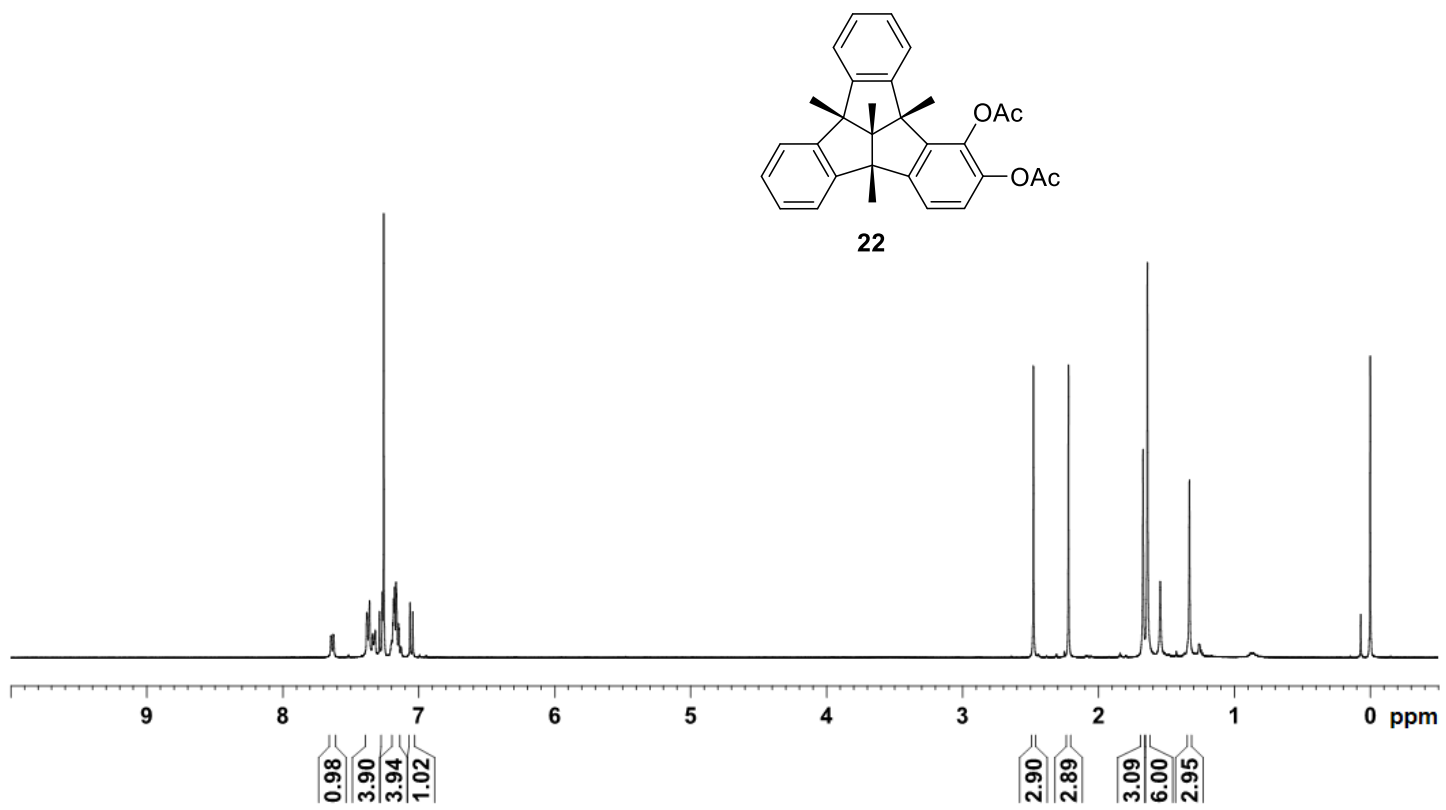

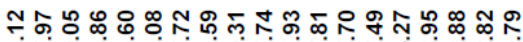

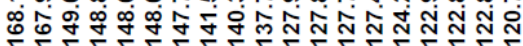
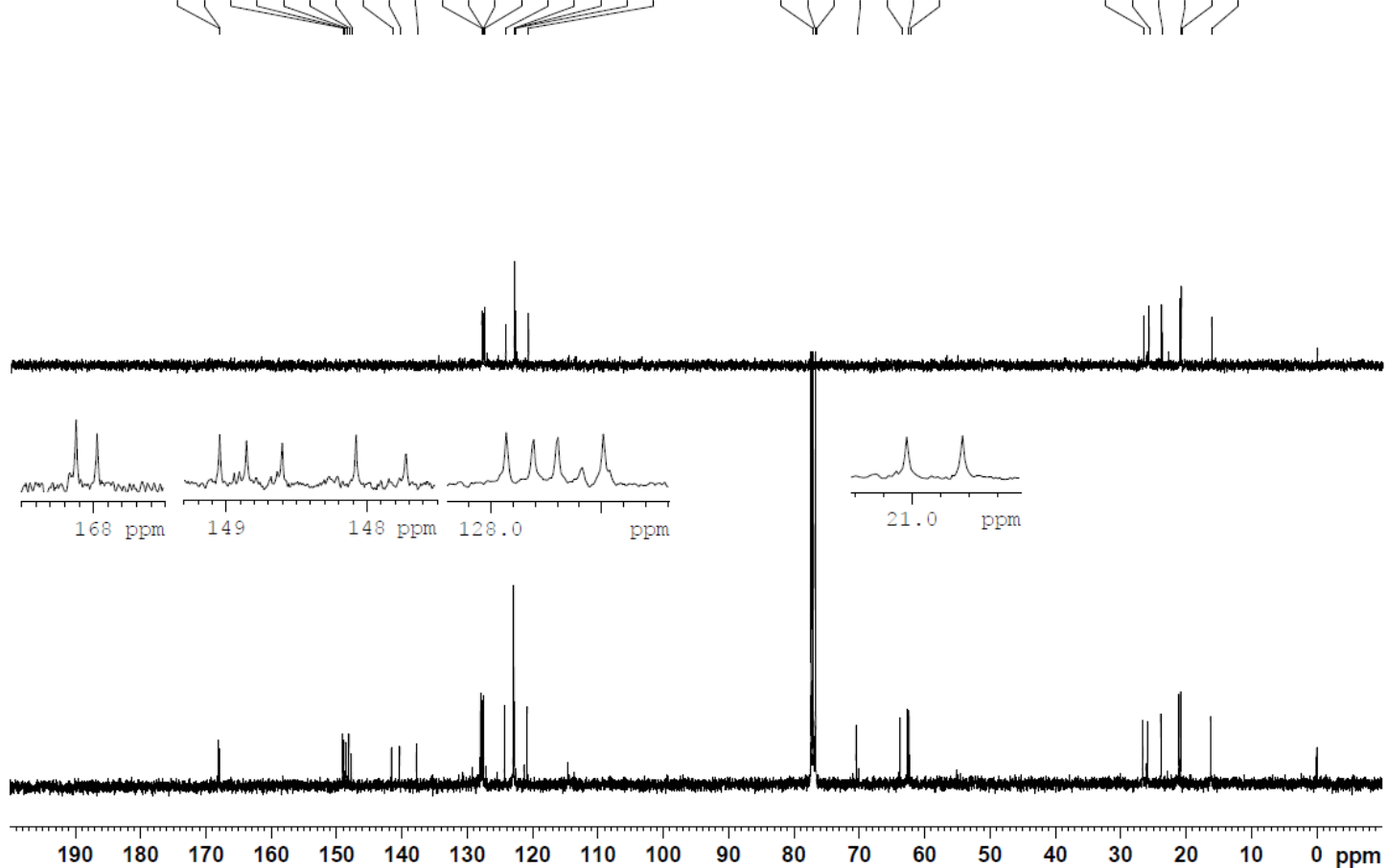
${ }^{1} \mathrm{H}\left(400 \mathrm{MHz}, \mathrm{CDCl}_{3}\right)$ and ${ }^{13} \mathrm{C} \mathrm{NMR}\left(100 \mathrm{MHz}, \mathrm{CDCl}_{3}\right)$ spectra of 23

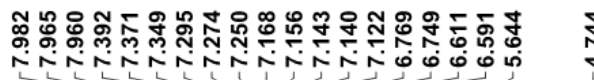
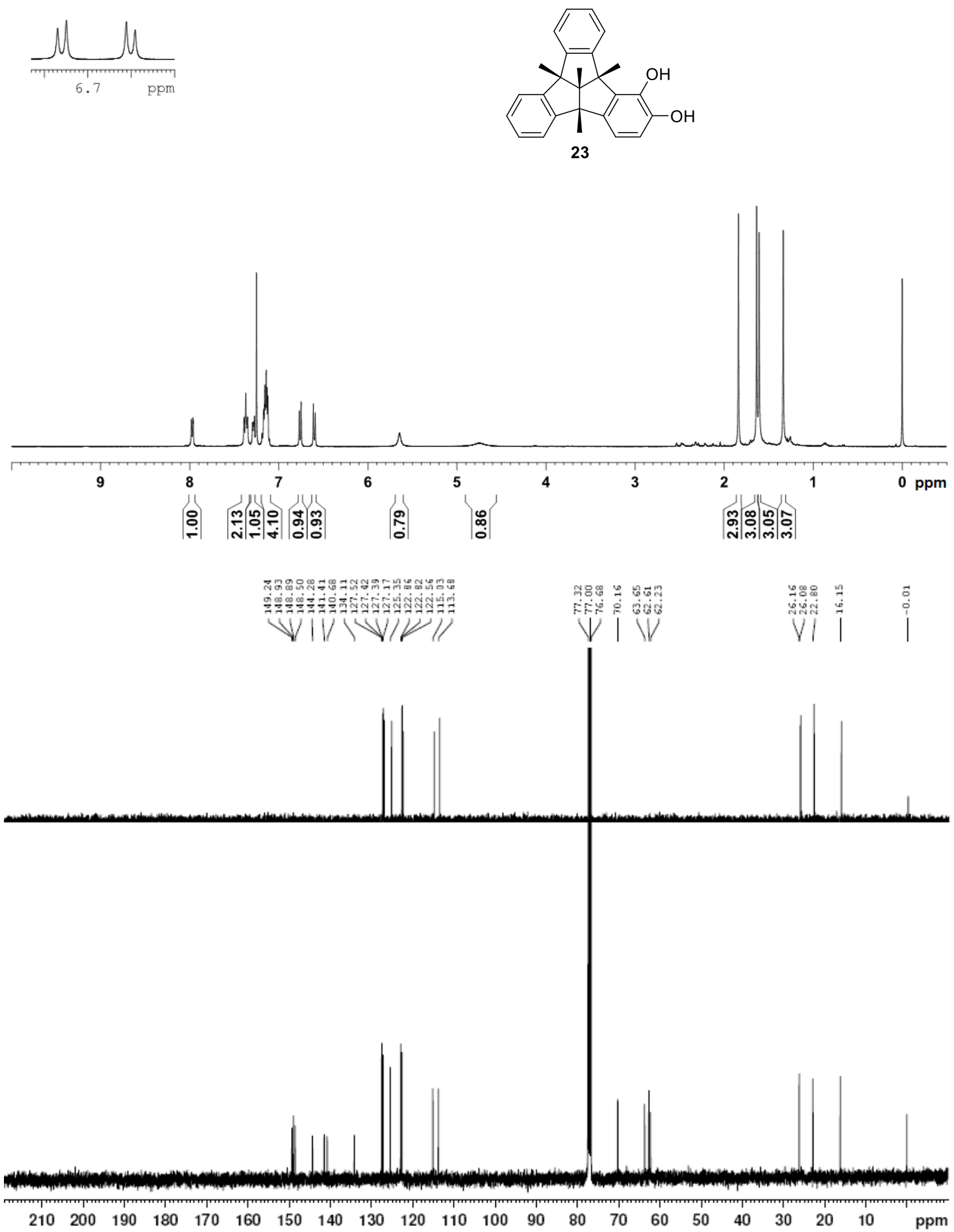
${ }^{1} \mathrm{H}(400 \mathrm{MHz}, \mathrm{CDCl} 3),{ }^{13} \mathrm{C} \mathrm{NMR}(100 \mathrm{MHz}, \mathrm{CDCl} 3)$, and $\mathrm{HMBC}$ spectra of 24

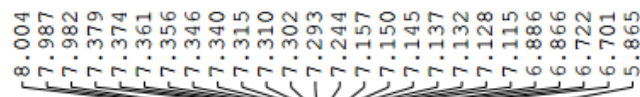

$\stackrel{\infty}{\stackrel{2}{~}}$

ivi
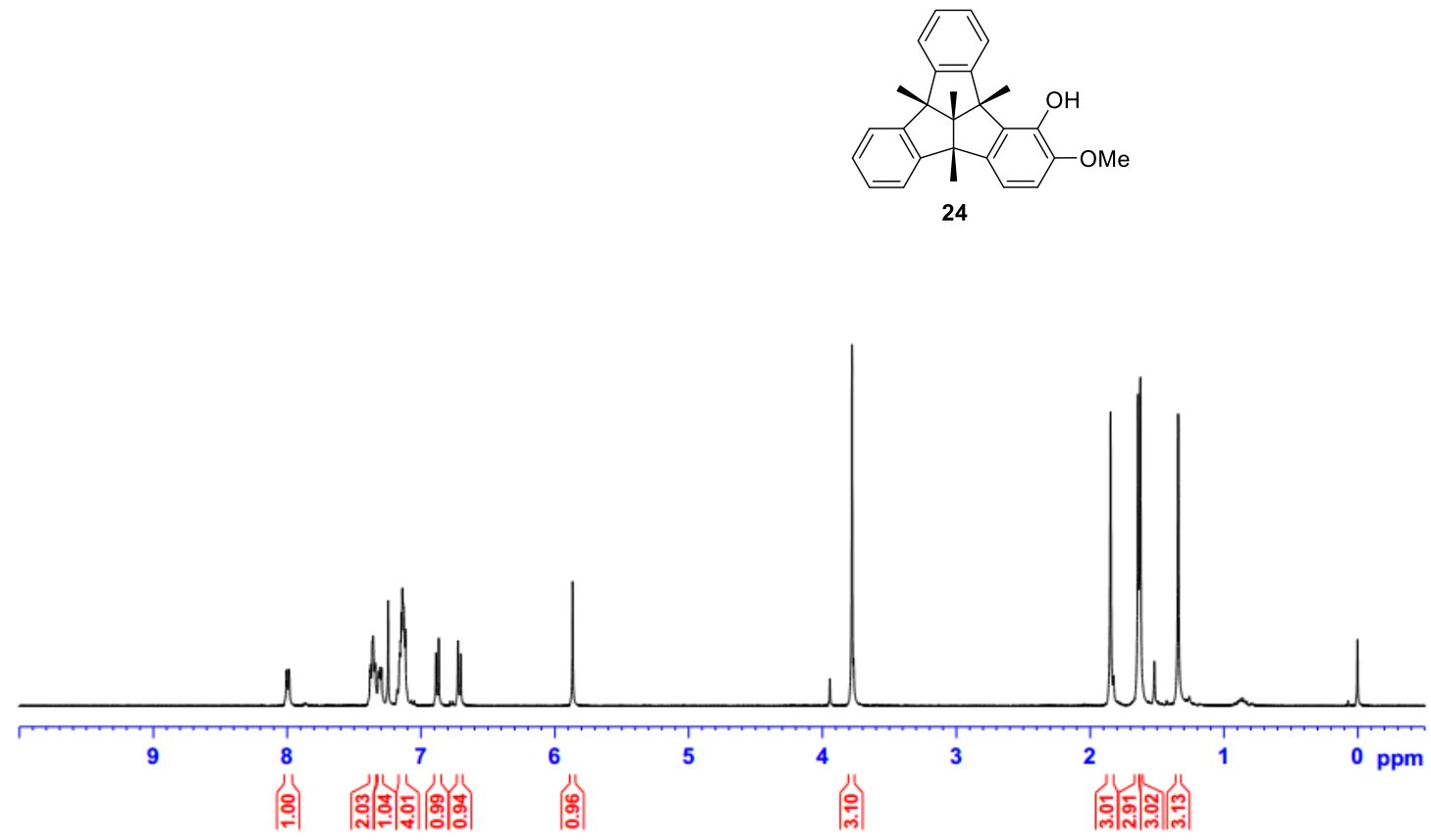

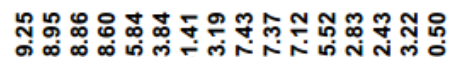

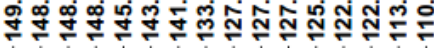

న్లె 下रํํํํำ

సิ \%ㅇำ

V VI

สู่ส่อ

VI
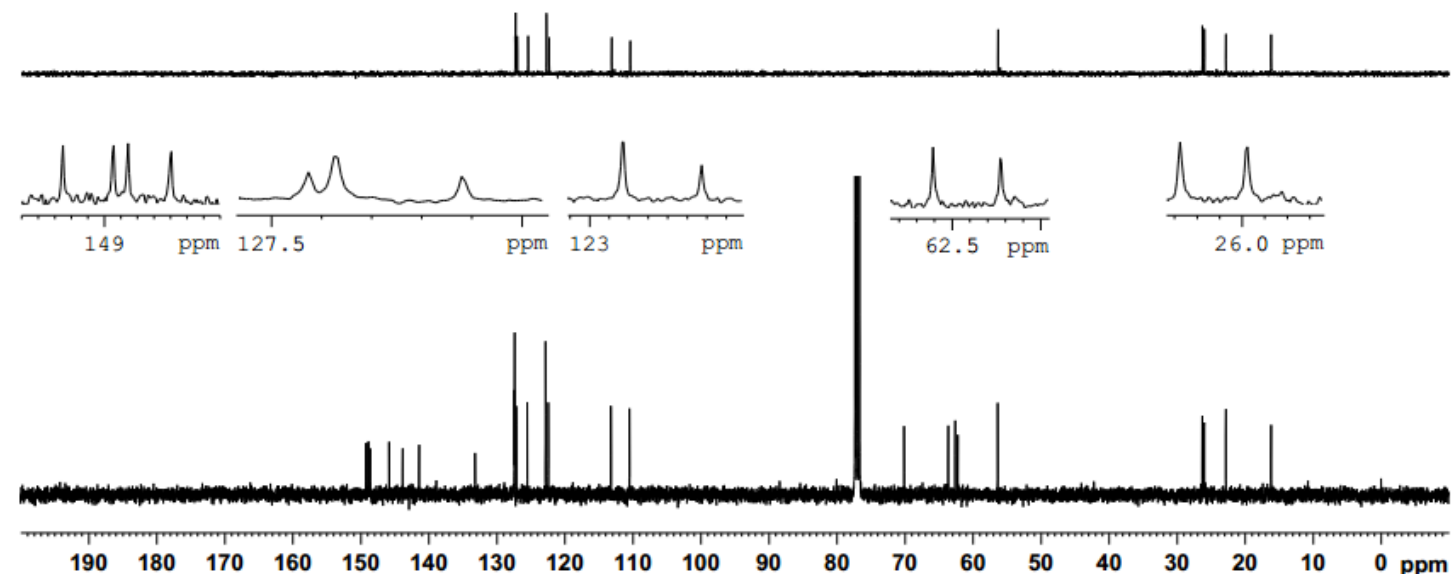

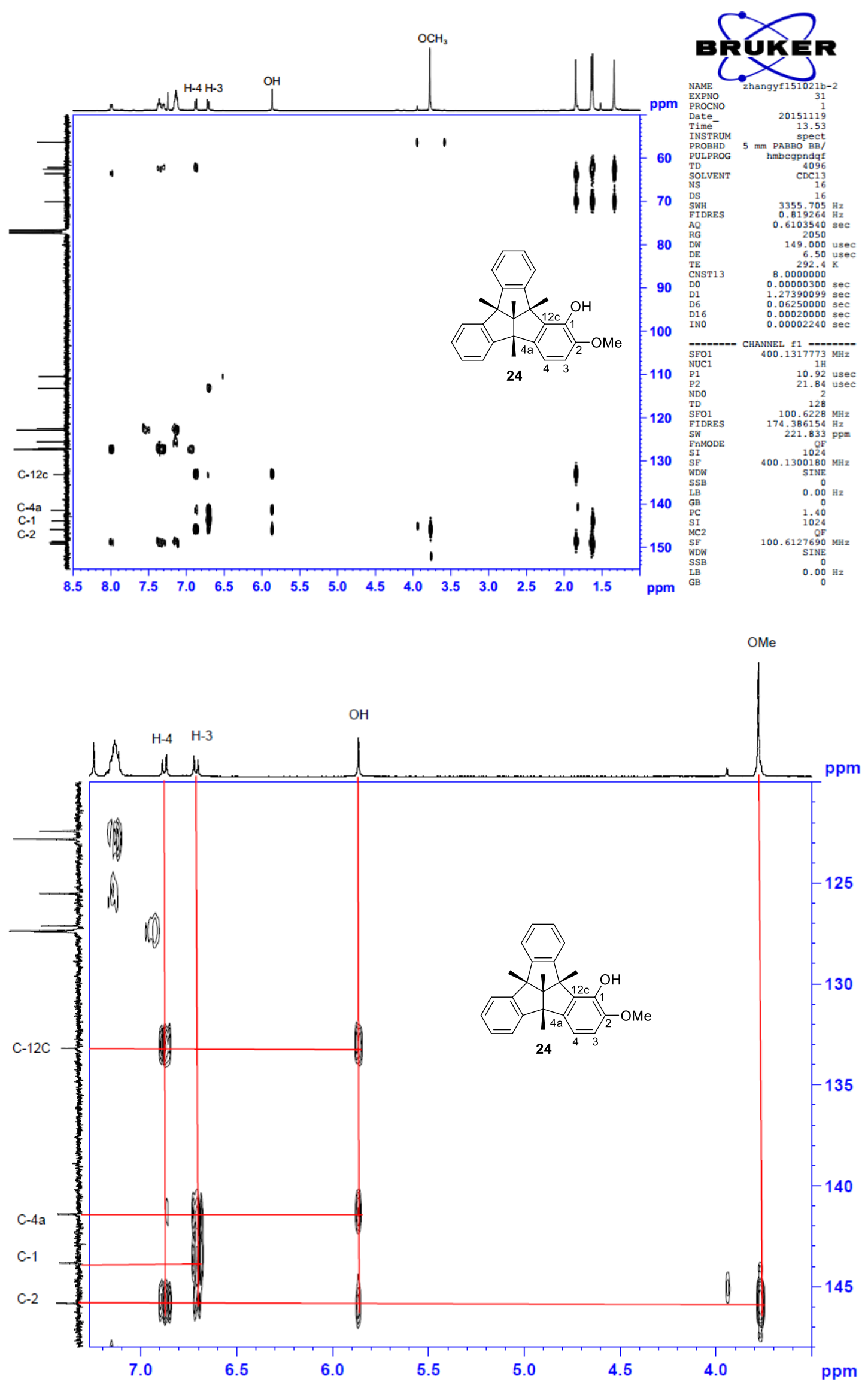
${ }^{1} \mathrm{H}\left(400 \mathrm{MHz}, \mathrm{CDCl}_{3}\right)$ and ${ }^{13} \mathrm{C}$ NMR $\left(100 \mathrm{MHz}, \mathrm{CDCl}_{3}\right)$ spectra of 25
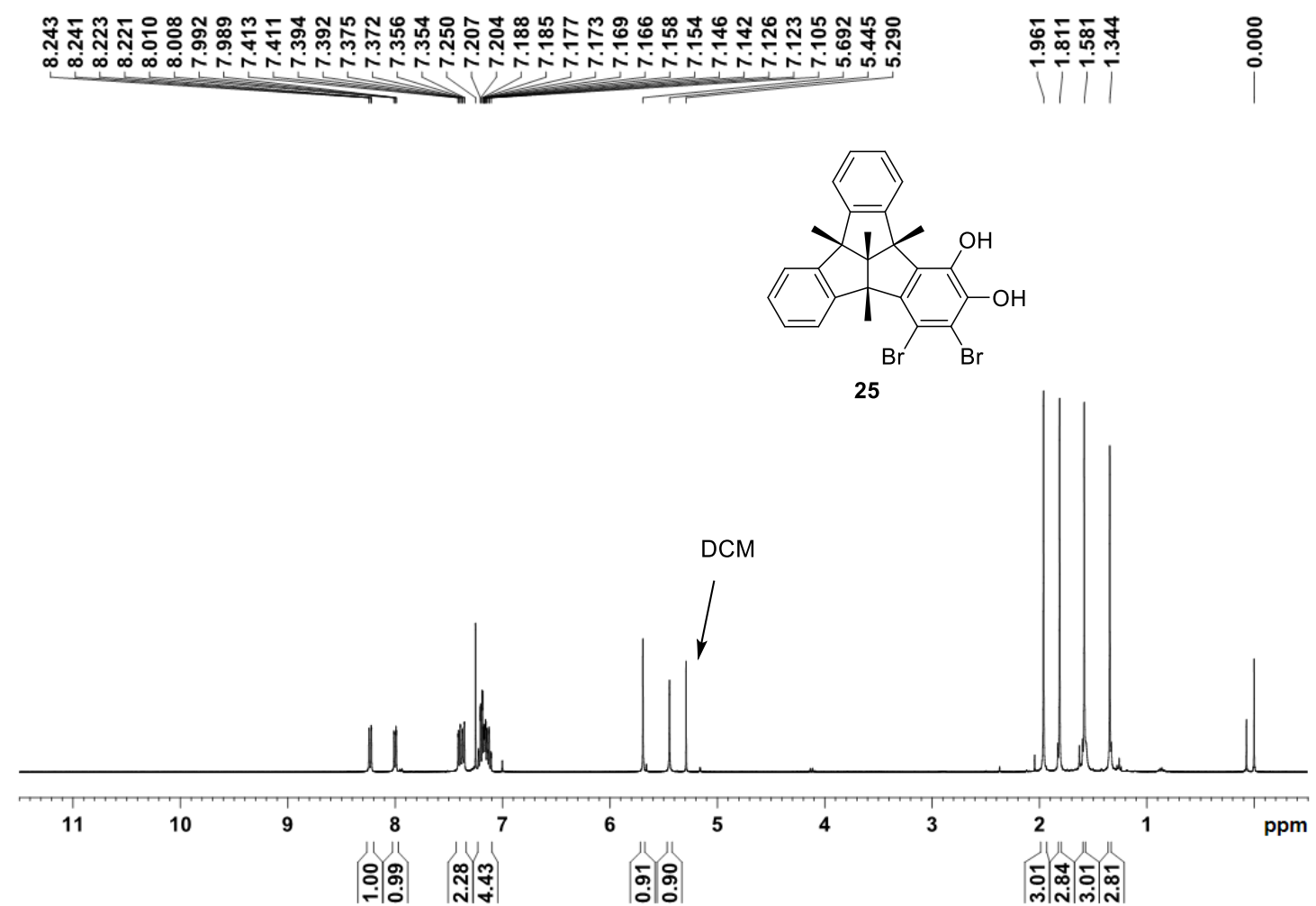

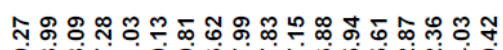

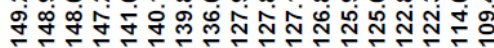

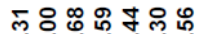

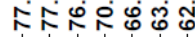

웅 운궁유

พัก กั่

1/1
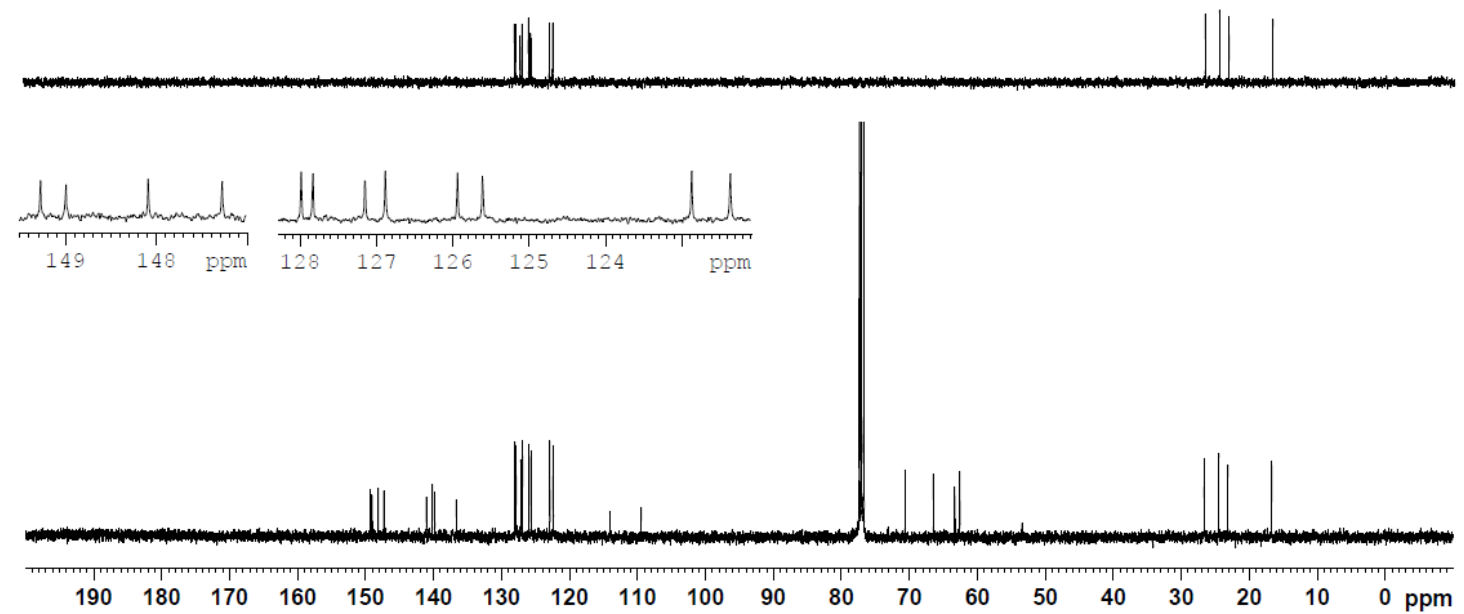
${ }^{1} \mathrm{H}\left(400 \mathrm{MHz}, \mathrm{CDCl}_{3}\right)$ and ${ }^{13} \mathrm{C} \mathrm{NMR}\left(100 \mathrm{MHz}, \mathrm{CDCl}_{3}\right)$ spectra of $27 \mathbf{a}$

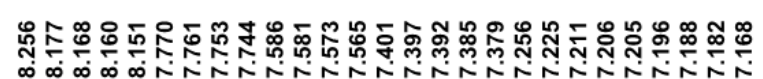
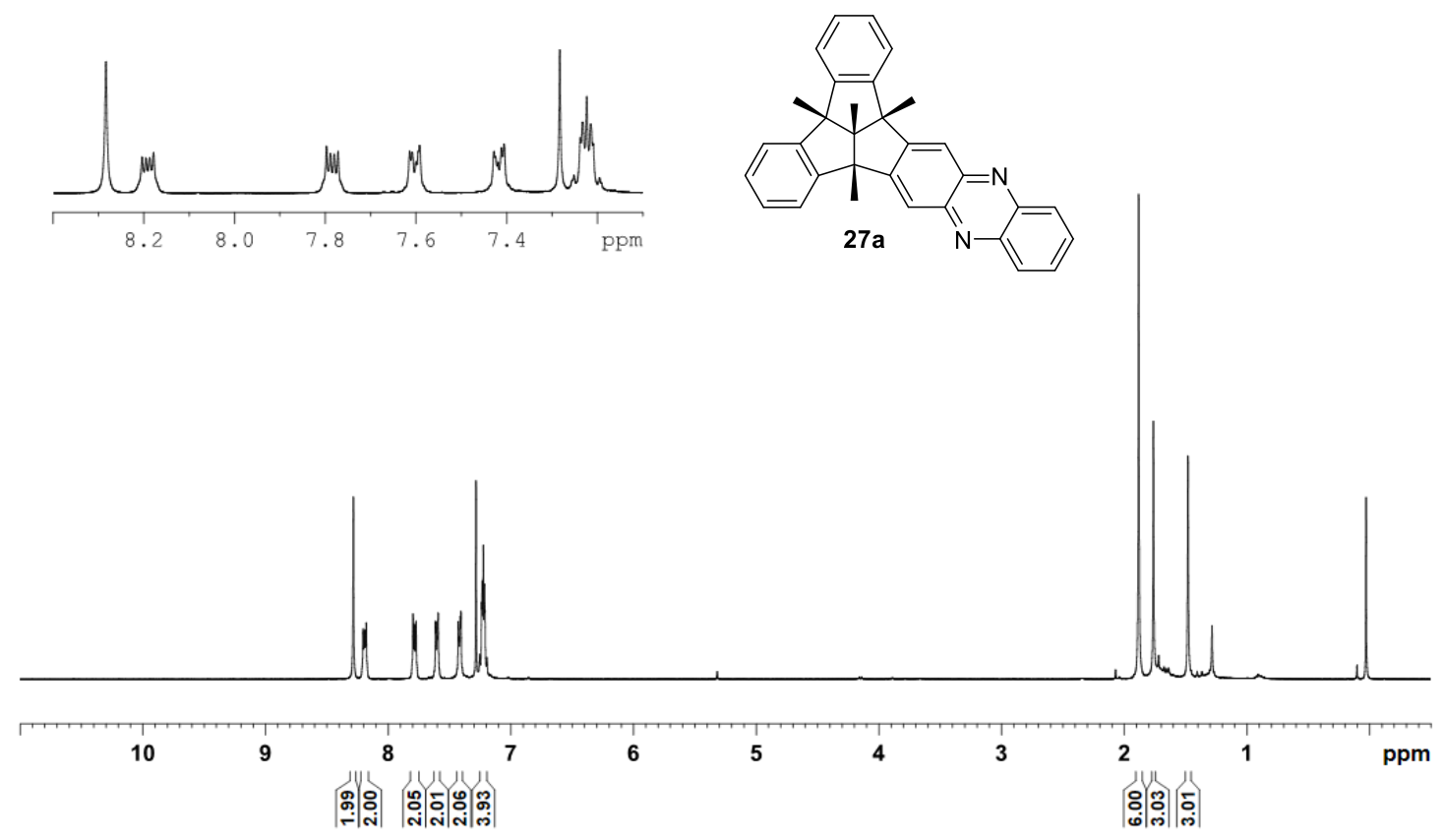

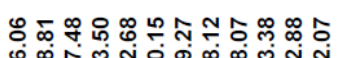

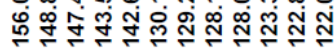
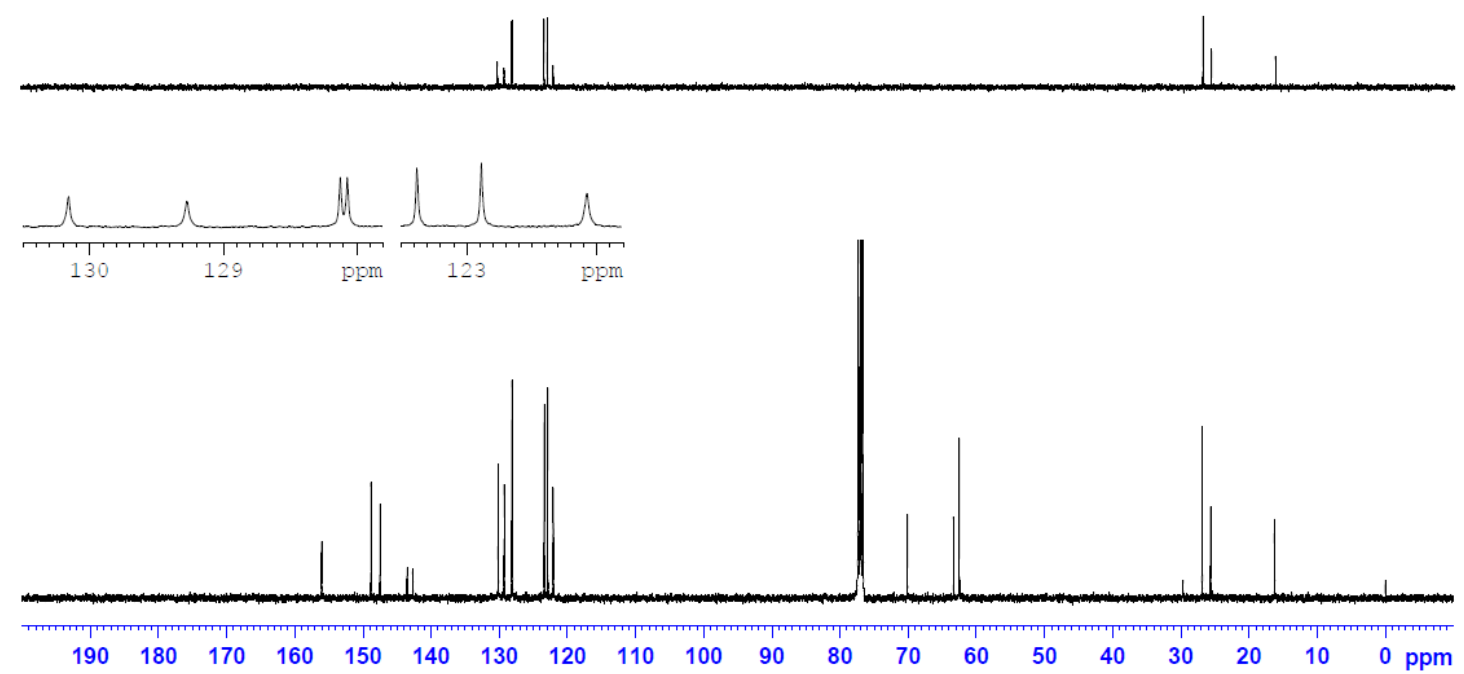
${ }^{1} \mathrm{H}\left(400 \mathrm{MHz}, \mathrm{CDCl}_{3}\right)$ and ${ }^{13} \mathrm{C} \mathrm{NMR}\left(100 \mathrm{MHz}, \mathrm{CDCl}_{3}\right)$ spectra of 27b

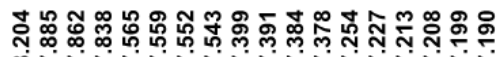

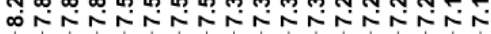
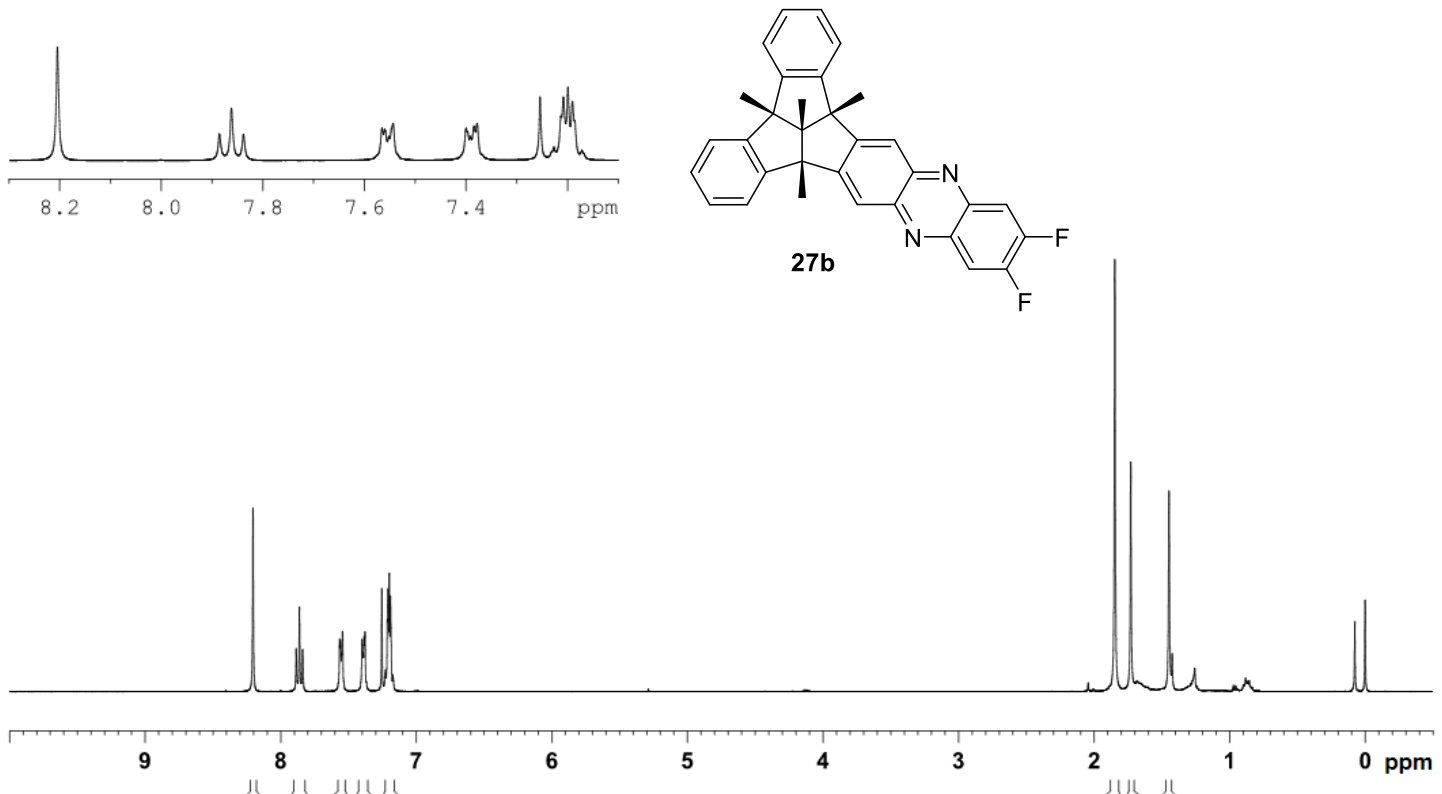
| :

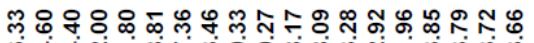

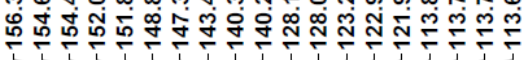

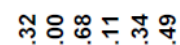
슈유ำ
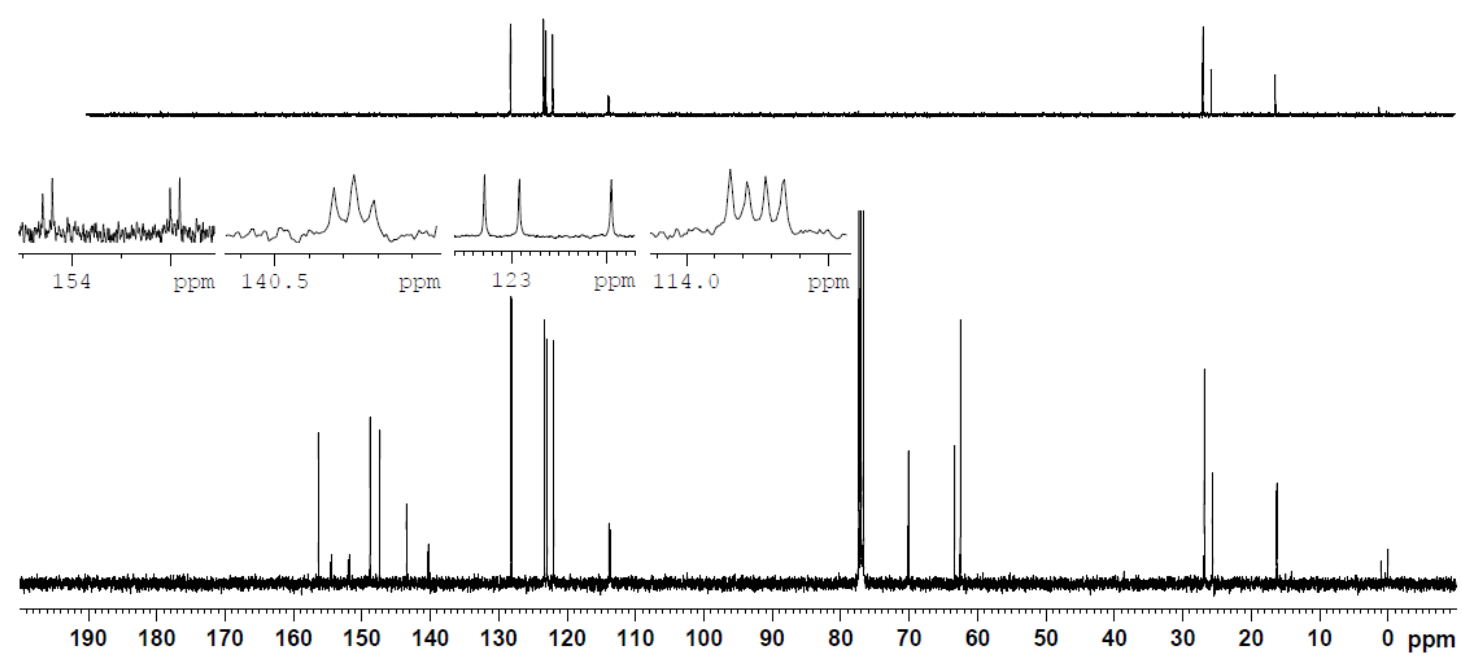
${ }^{1} \mathrm{H}\left(400 \mathrm{MHz}, \mathrm{CDCl}_{3}\right)$ and ${ }^{13} \mathrm{C}$ NMR $\left(100 \mathrm{MHz}, \mathrm{CDCl}_{3}\right)$ spectra of 27c

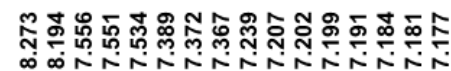

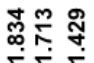
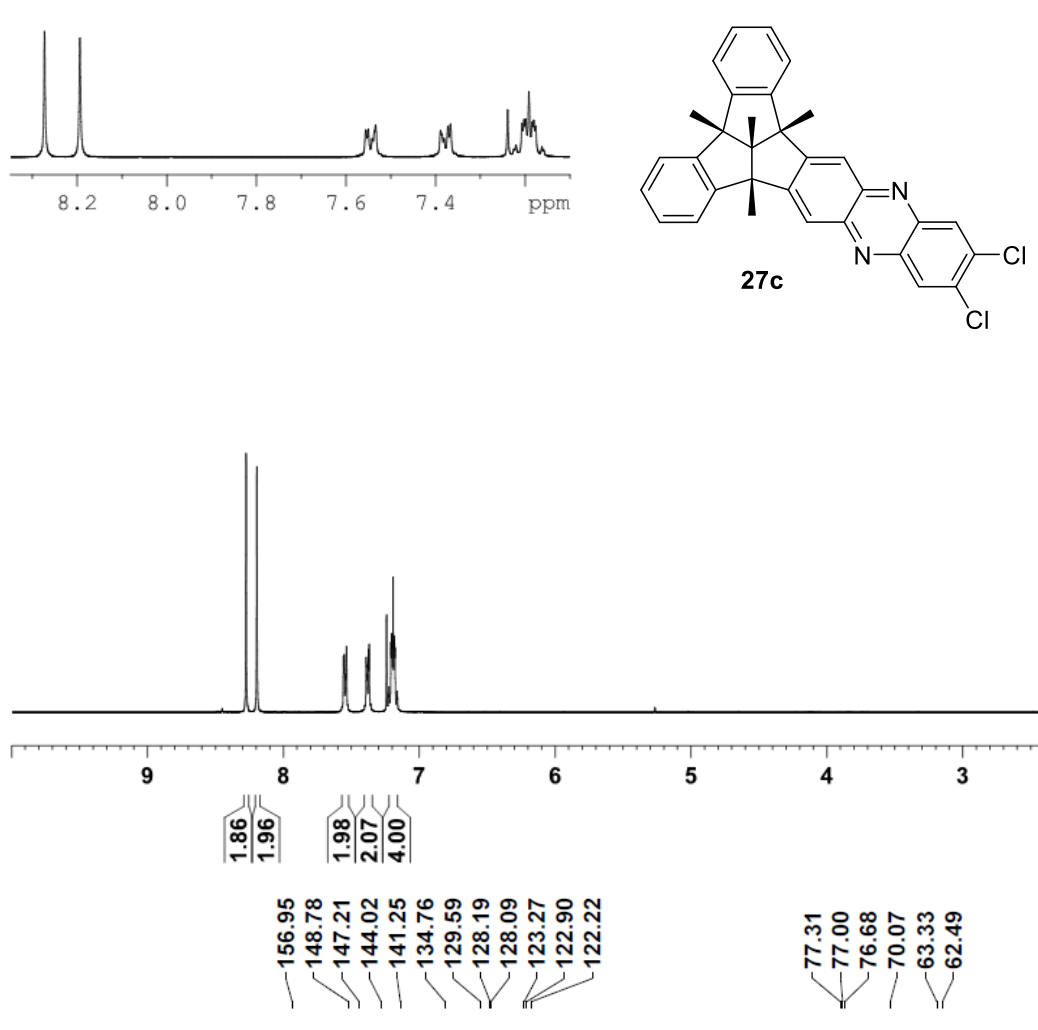

ஸึ่
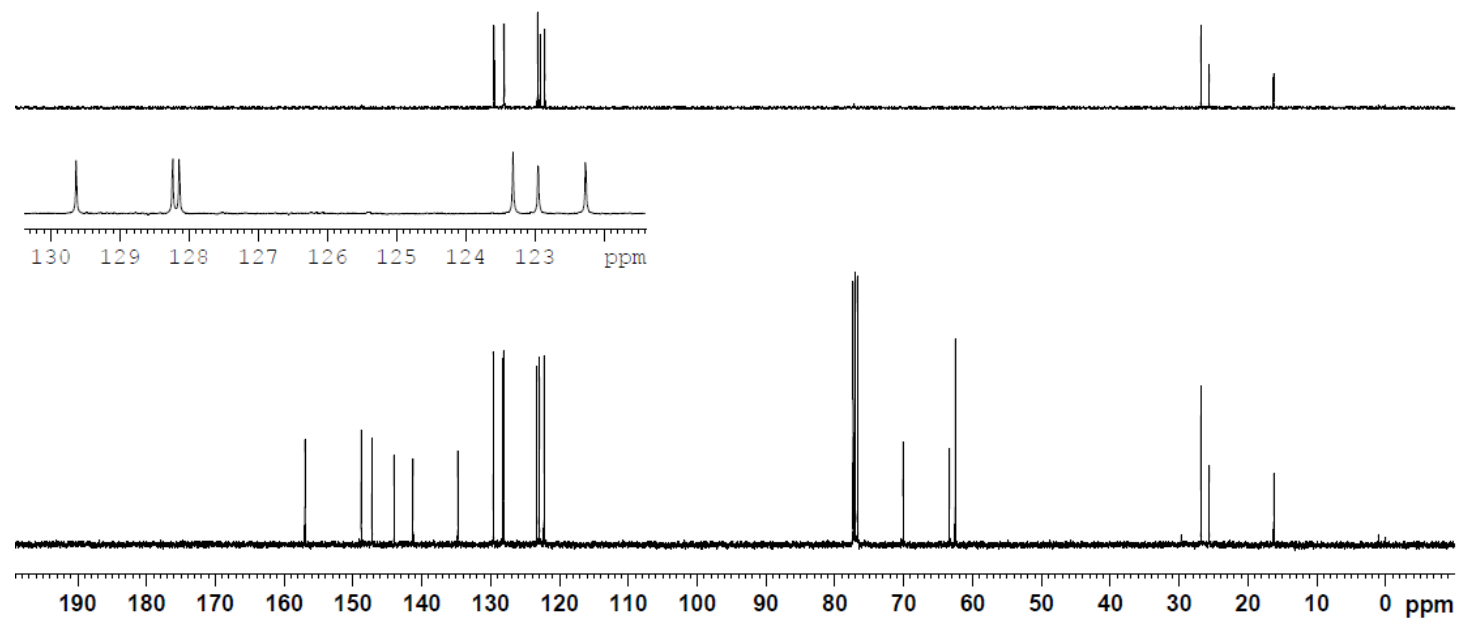
${ }^{1} \mathrm{H}\left(400 \mathrm{MHz}, \mathrm{CDCl}_{3}\right)$ and ${ }^{13} \mathrm{C} \mathrm{NMR}\left(100 \mathrm{MHz}, \mathrm{CDCl}_{3}\right)$ spectra of 27d

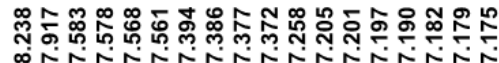
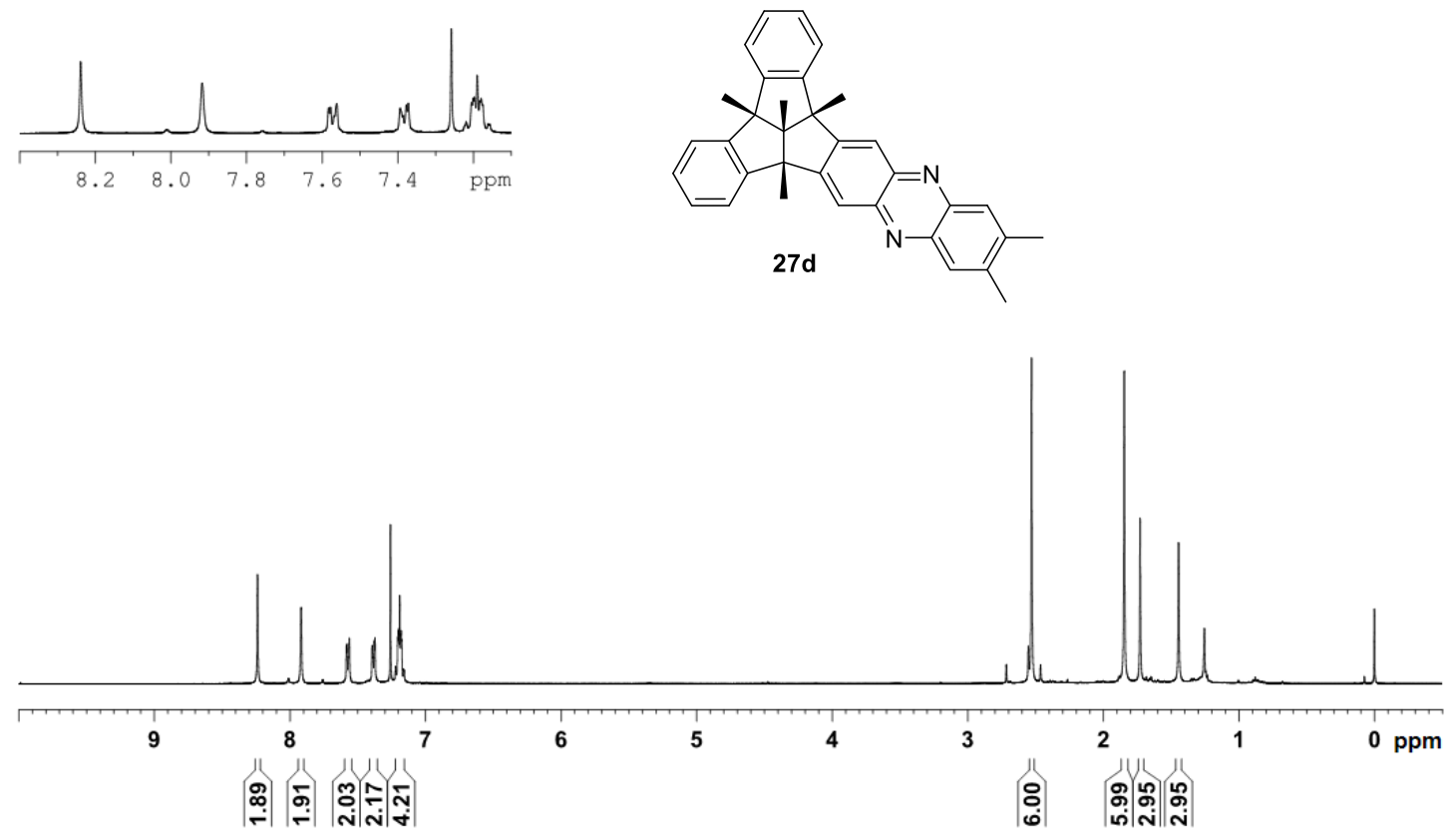

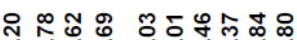

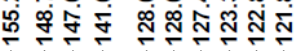

ำ 웅ำ

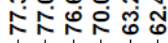

๘

W I

ผู่ ผ่อ

/ 11
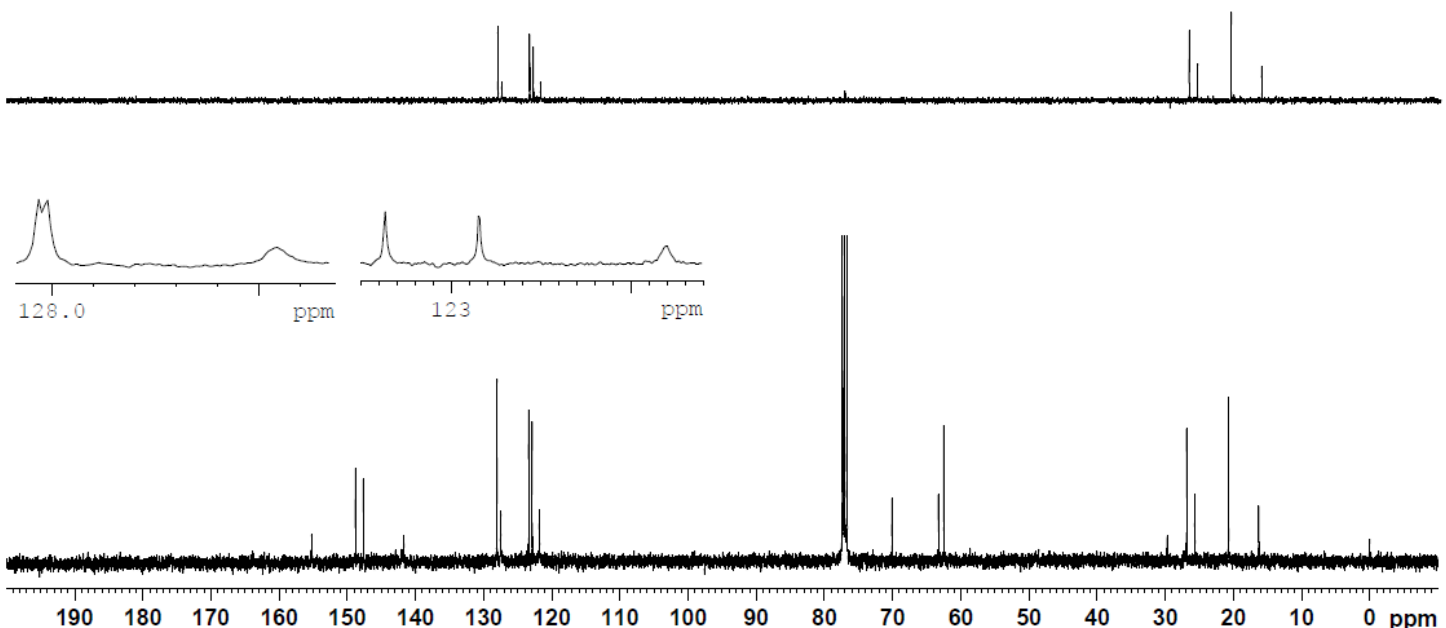
${ }^{1} \mathrm{H}\left(400 \mathrm{MHz}, \mathrm{CDCl}_{3}\right)$ and ${ }^{13} \mathrm{C} \mathrm{NMR}\left(100 \mathrm{MHz}, \mathrm{CDCl}_{3}\right)$ spectra of $27 \mathbf{e}$

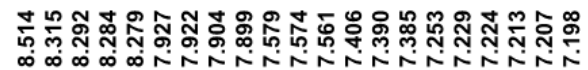
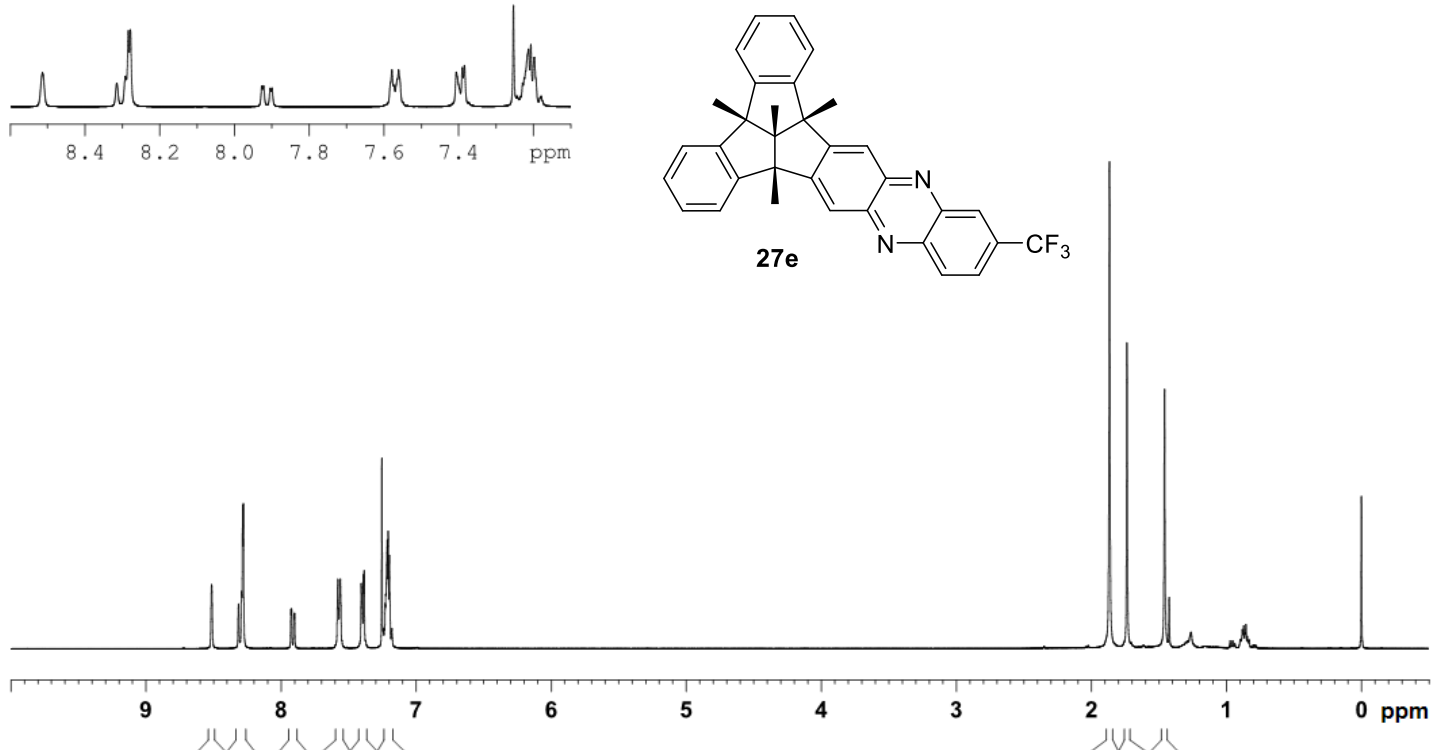

:

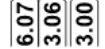
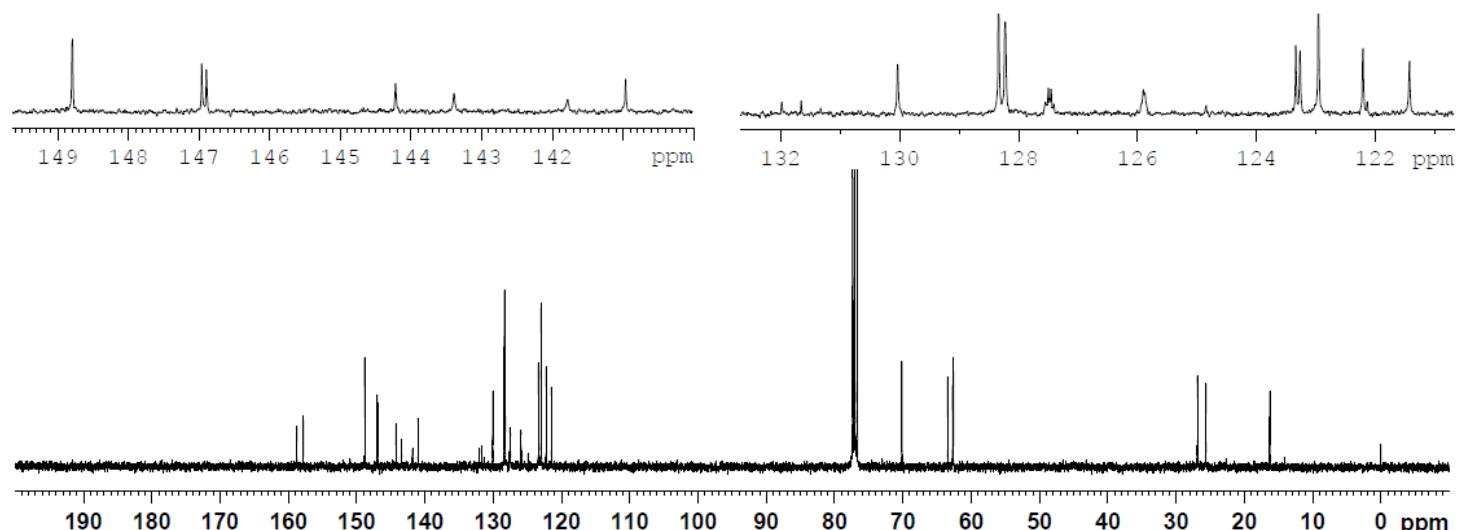
${ }^{1} \mathrm{H}\left(400 \mathrm{MHz}, \mathrm{CDCl}_{3}\right)$ and ${ }^{13} \mathrm{C}$ NMR $\left(100 \mathrm{MHz}, \mathrm{CDCl}_{3}\right)$ spectra of $27 \mathbf{f}$

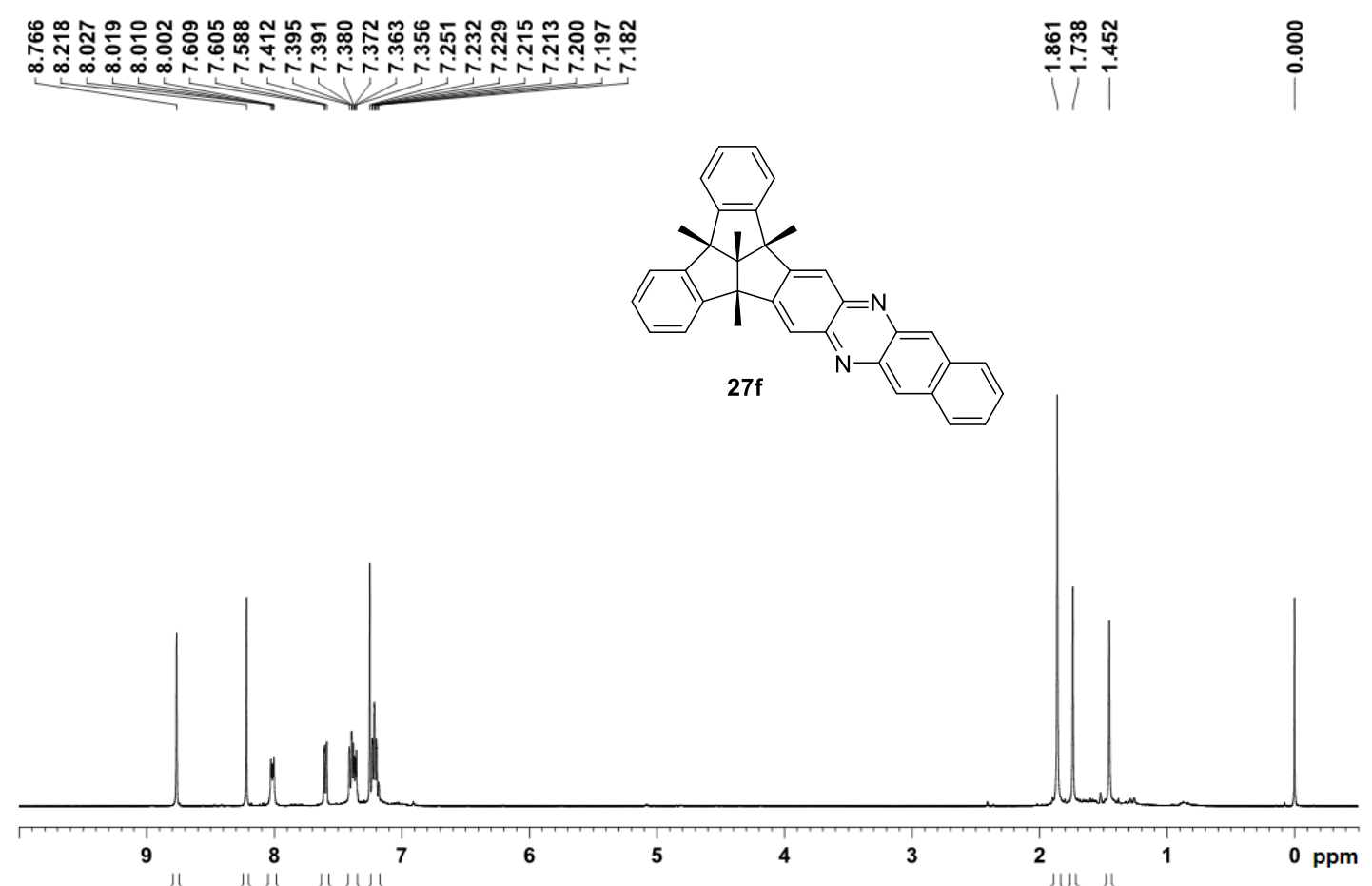

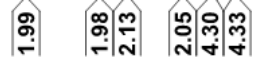

:

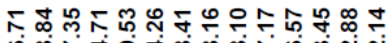

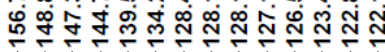

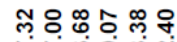

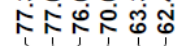

ஸ્仓
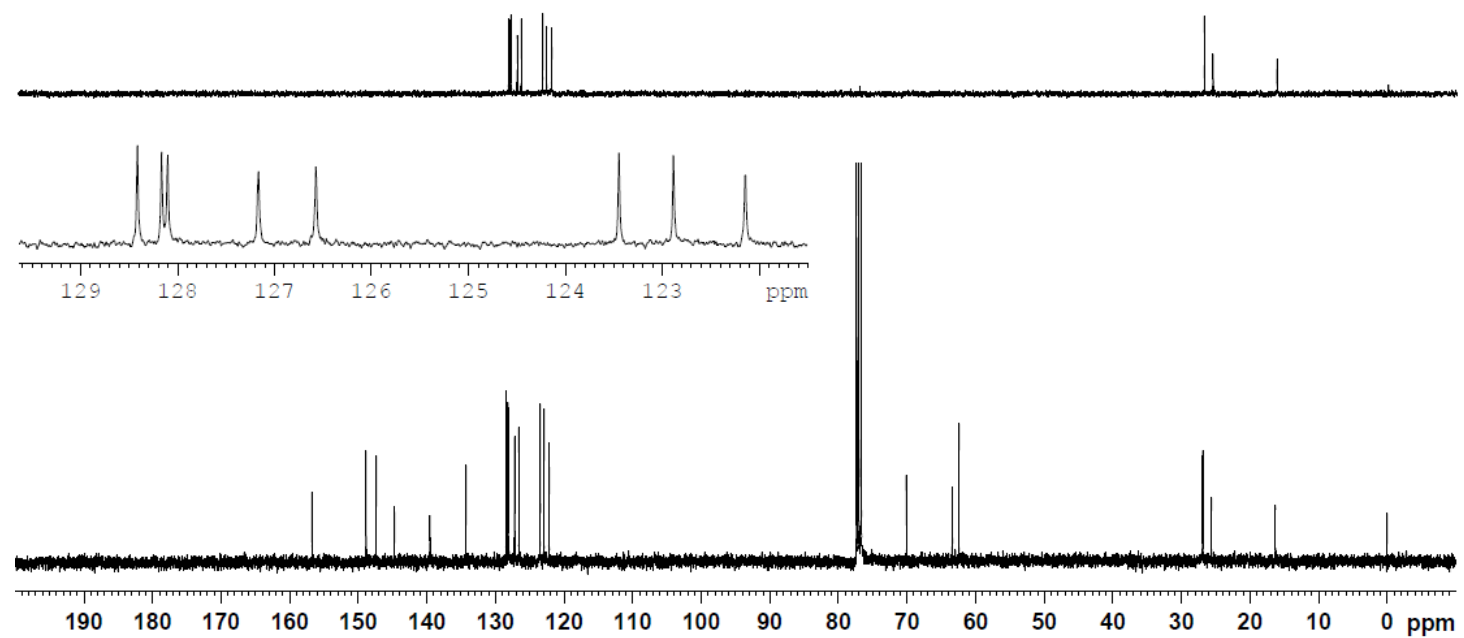
${ }^{1} \mathrm{H}\left(400 \mathrm{MHz}, \mathrm{CDCl}_{3}\right)$ and ${ }^{13} \mathrm{C} \mathrm{NMR}\left(100 \mathrm{MHz}, \mathrm{CDCl}_{3}\right)$ spectra of 30

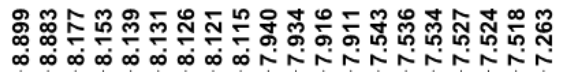
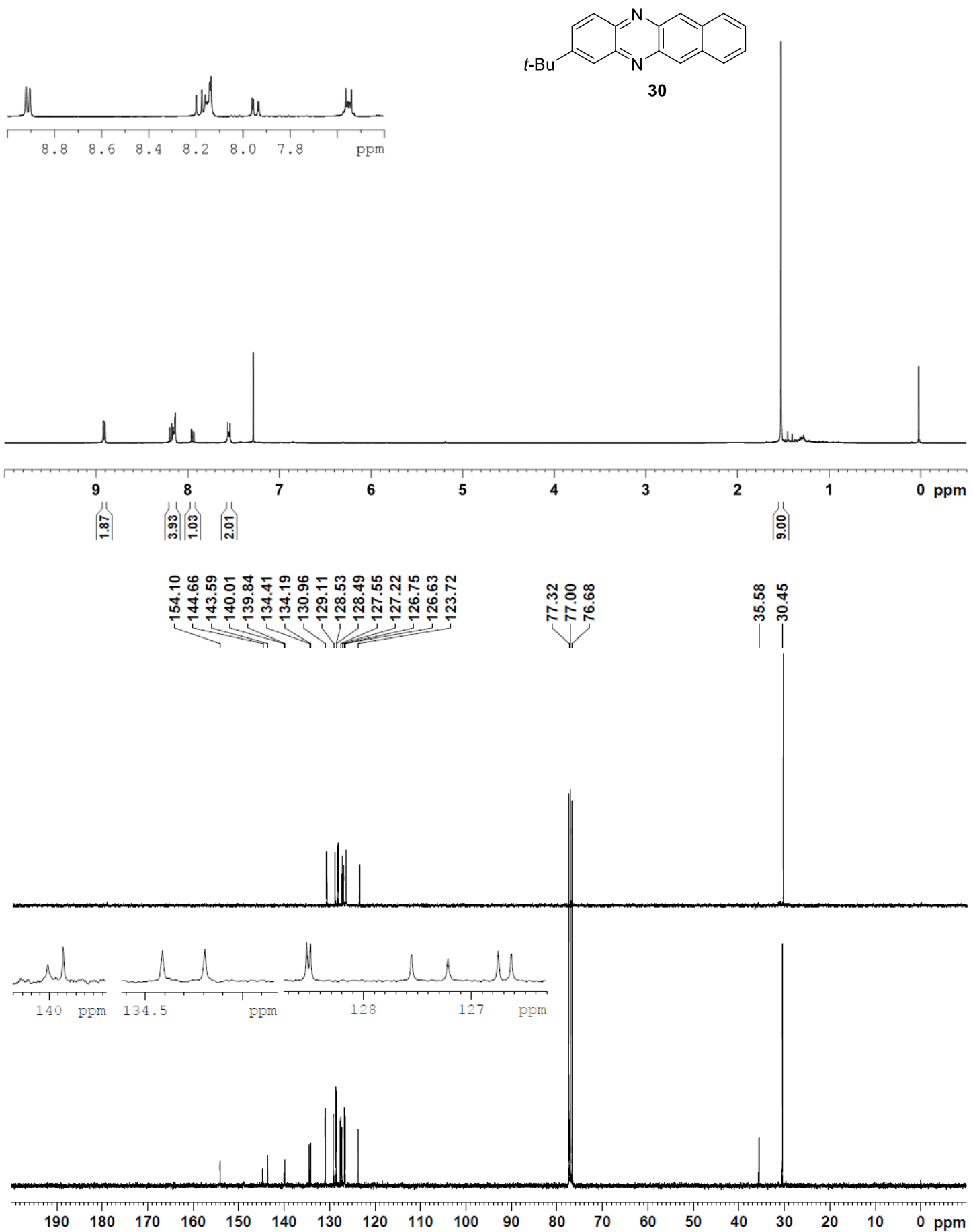
${ }^{1} \mathrm{H}\left(400 \mathrm{MHz}, \mathrm{CDCl}_{3}\right)$ and ${ }^{13} \mathrm{C}$ NMR $\left(100 \mathrm{MHz}, \mathrm{CDCl}_{3}\right)$ spectra of 32

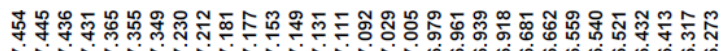

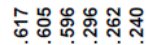

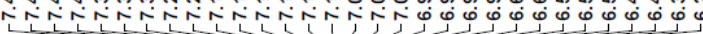

$\sqrt{1 / 2}$
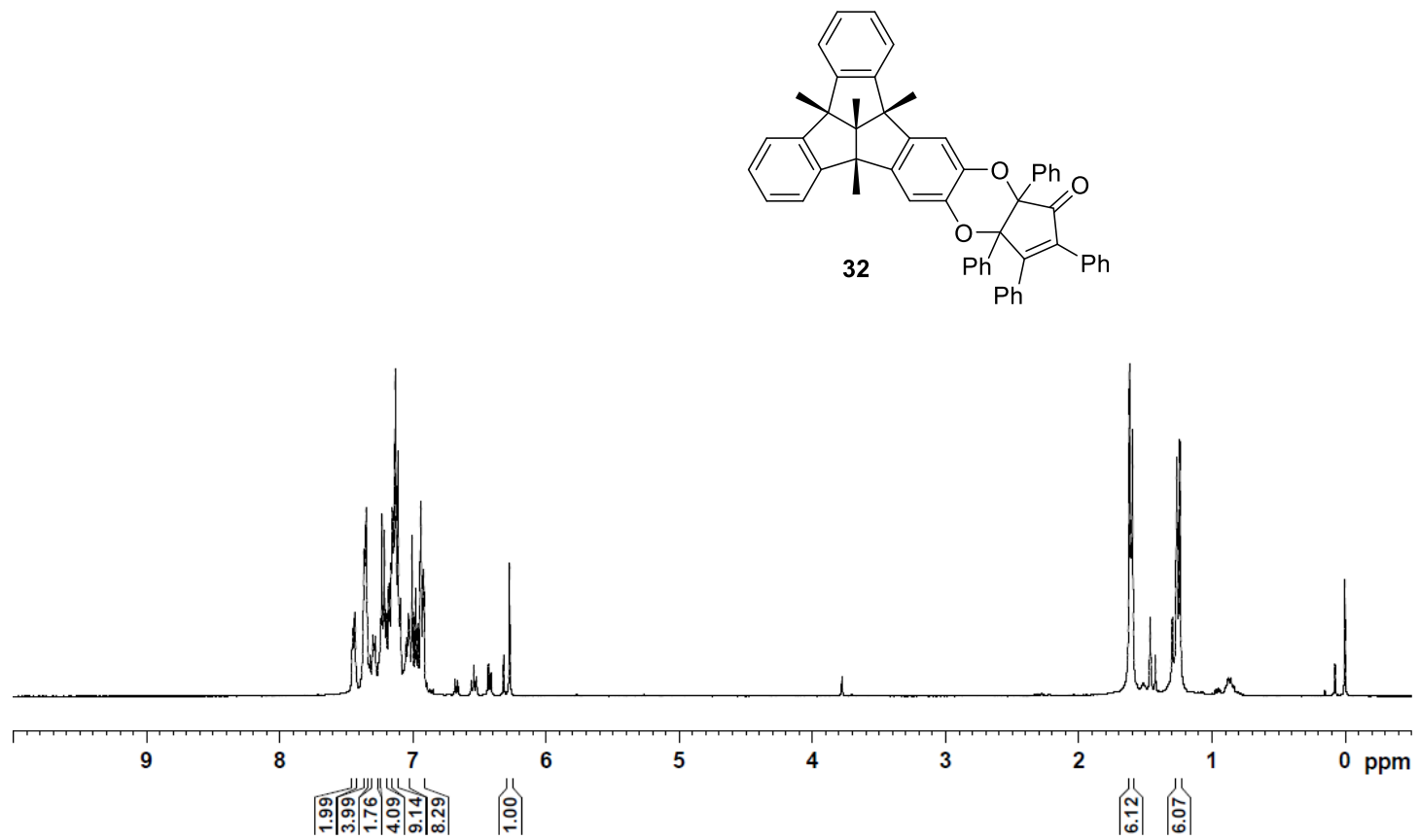

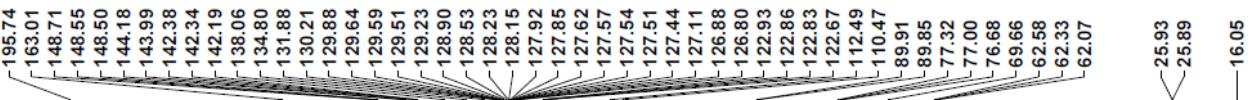

11.
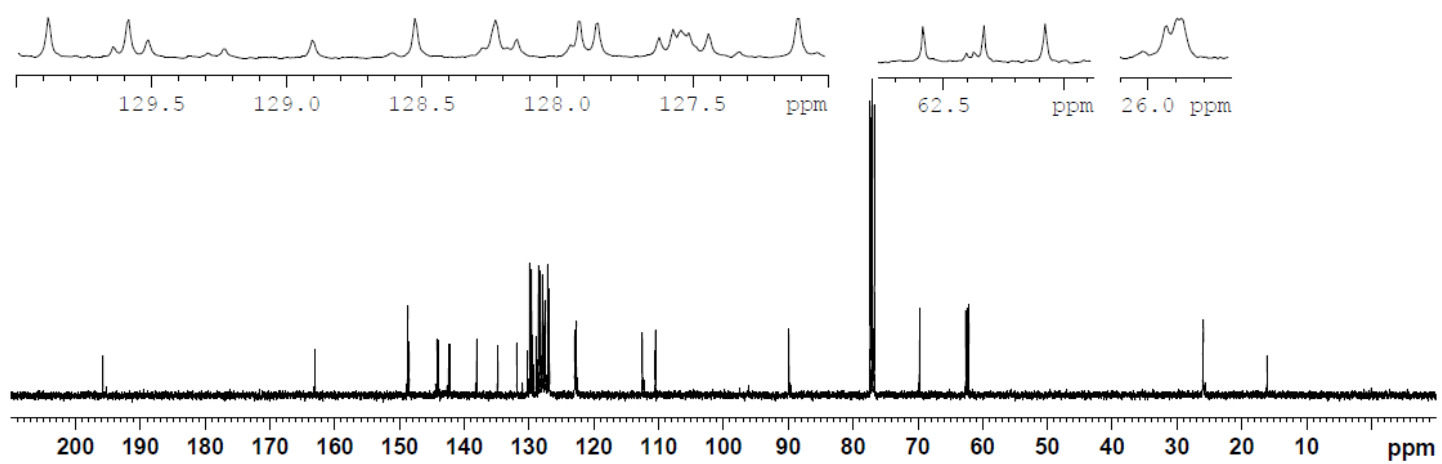

Spectrum has been recorded from a mixture of stereoisomers that cannot be separated by flash column chromatography. 
(2) X-ray crystallographic data of $\mathrm{rac}-\mathbf{1 4}, \mathbf{1 7} \cdot$ EtOAc, 17· THF, $23 \cdot$ acetone, 18, 27b, 27c, and 32 (endo-anti-stereoisomer).

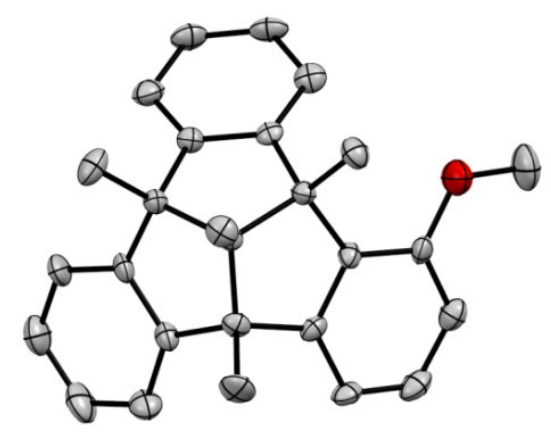

Figure S1. Crystal structure of rac-14 (ORTEP diagram, ellipsoids are shown at 30\% probability level; hydrogen atoms are omitted for clarity).

X-ray crystal data for $\mathbf{1 4}$ from ethyl acetate/petroleum ether: $\mathrm{C}_{27} \mathrm{H}_{26} \mathrm{O} ; M=366.48$; orthorhombic; $a=20.9274(10) \AA, b=15.0038(6) \AA, c=6.3388(4) \AA ; \alpha=90.00^{\circ}, \beta=$ $90.00^{\circ}, \gamma=90.00^{\circ} ; V=1990.33(10) \AA^{3}$; space group $P n 21 \mathrm{a} ; Z=4 ; \rho_{\text {calcd }}=1.223 \mathrm{Mg}$ $\mathrm{m}^{-3} ; T=291.73(10) \mathrm{K} ; \lambda(\mathrm{Mo} \mathrm{K} \alpha)=0.71073 \AA ; 3086$ reflections collected;

1967 independent reflections; $R_{\text {int }}=0.0352 ; \mathrm{R} 1=0.0816$, wR2 $=0.2159[\mathrm{I} \geqslant 2 \sigma(\mathrm{I})]$. CCDC-1056870 contains the supplementary crystallographic data for 14 .

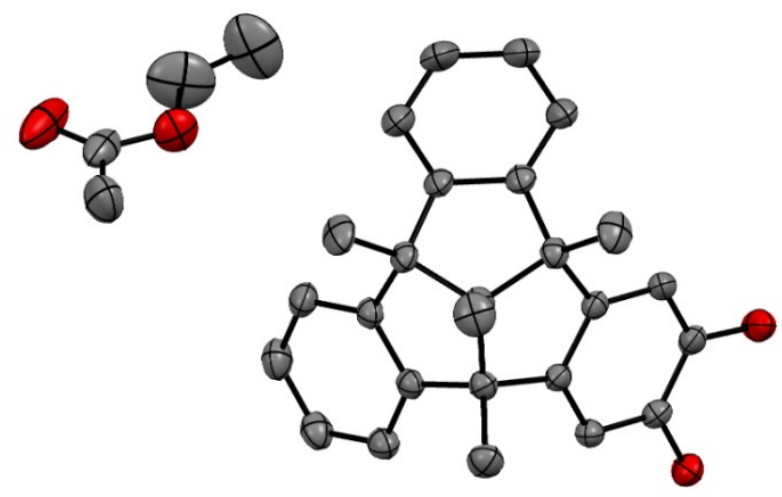

Figure S2. Crystal structure of 17·EtOAc (ORTEP diagram, ellipsoids are shown at $30 \%$ probability level; hydrogen atoms are omitted for clarity).

X-ray crystal data for 17. EtOAc from ethyl acetate/petroleum ether:

$\mathrm{C}_{26} \mathrm{H}_{24} \mathrm{O}_{2} \cdot \mathrm{C}_{4} \mathrm{H}_{8} \mathrm{O}_{2} ; M=456.55$; monoclinic; $a=9.8861(5) \AA, b=23.7464(14) \AA, c=$ $10.5247(15) \AA ; \alpha=90.00^{\circ}, \beta=96.553(7)^{\circ}, \gamma=90.00^{\circ} ; V=2454.6(4) \AA^{3}$; space group $P 21 / \mathrm{c} ; Z=4 ; \rho_{\text {calcd }}=1.235 \mathrm{Mg} \mathrm{m}^{-3} ; T=295.6(2) \mathrm{K} ; \lambda(\mathrm{Mo} \mathrm{K} \alpha)=0.7107 \AA ; 5570$ reflections collected; 2818 independent reflections; $R_{\text {int }}=0.0691 ; \mathrm{R} 1=0.0769$, wR2 $=$ $0.1774[\mathrm{I} \geq 2 \sigma(\mathrm{I})]$. CCDC-1028568 contains the supplementary crystallographic data for $\mathbf{1 7} \cdot$ EtOAc. 


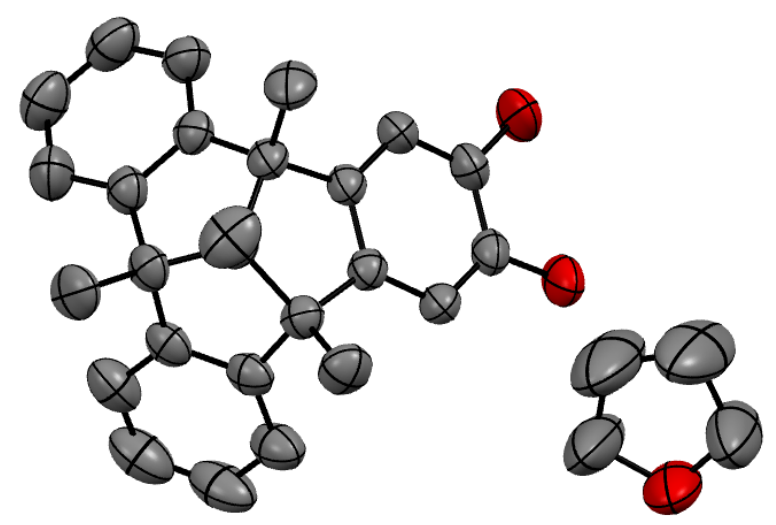

Figure S3. Crystal structure of $\mathbf{1 7} \cdot$ THF (ORTEP diagram, ellipsoids are shown at $30 \%$ probability level; hydrogen atoms are omitted for clarity).

X-ray crystal data for $\mathbf{1 7} \cdot$ THF from tetrahydrofuran: $\mathrm{C}_{26} \mathrm{H}_{24} \mathrm{O}_{2} \cdot \mathrm{C}_{4} \mathrm{H}_{4} \mathrm{O} ; M=436.52$; monoclinic; $a=17.9484(5) \AA, b=8.6969(2) \AA, c=16.5941(5) \AA ; \alpha=90.00^{\circ}, \beta=$ $109.720(3)^{\circ}, \gamma=90.00^{\circ} ; V=2438.35(13) \AA^{3}$; space group $P 21 / \mathrm{c} ; Z=4 ; \rho_{\text {calcd }}=1.189$ $\mathrm{Mg} \mathrm{m}^{-3} ; T=298.5(10) \mathrm{K} ; \lambda(\mathrm{Cu} \mathrm{K} \alpha)=1.5418 \AA ; 4562$ reflections collected; 3924 independent reflections; $R_{\text {int }}=0.0192 ; \mathrm{R} 1=0.0618$, wR2 $=0.1839[\mathrm{I} \geq 2 \sigma(\mathrm{I})]$. CCDC-1028569 contains the supplementary crystallographic data for $\mathbf{1 7} \cdot \mathrm{THF}$.

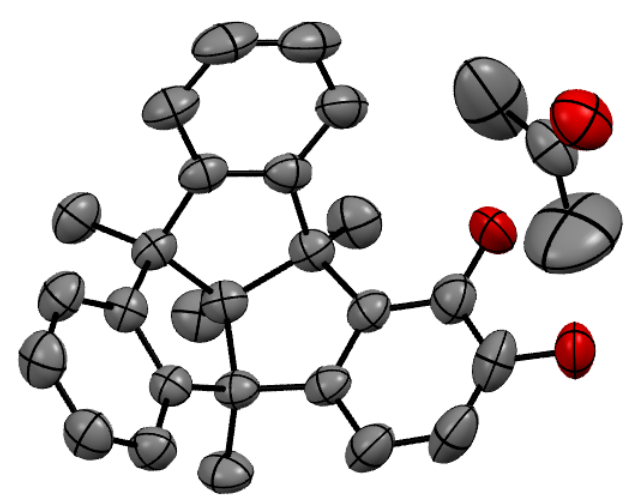

Figure S4. Crystal structure of $\mathrm{rac}-\mathbf{2 3} \cdot$ acetone (ORTEP diagram, ellipsoids are shown at $30 \%$ probability level; hydrogen atoms are omitted for clarity).

X-ray crystal data for $r a c-23$ acetone from acetone: $\mathrm{C}_{26} \mathrm{H}_{22} \mathrm{O}_{2} \cdot \mathrm{C}_{3} \mathrm{H}_{6} \mathrm{O} ; M=424.51$; monoclinic; $a=9.6832(6) \AA, b=15.9129(8) \AA, c=15.0454(9) \AA ; \alpha=90.00^{\circ}, \beta=$ $91.068(5)^{\circ}, \gamma=90.00^{\circ} ; V=2317.9(2) \AA^{3}$; space group $P 21 / \mathrm{n} Z=4 ; \rho_{\text {calcd }}=1.189 \mathrm{Mg}$ $\mathrm{m}^{-3} ; T=295(1) \mathrm{K} ; \lambda(\mathrm{Mo} \mathrm{K} \alpha)=0.7107 \AA ; 4503$ reflections collected; 3096 independent reflections; $R_{\text {int }}=0.0228 ; \mathrm{R} 1=0.0598$, wR2 $=0.1563[\mathrm{I} \geq 2 \sigma(\mathrm{I})]$. CCDC-1028570 contains the supplementary crystallographic data for $\mathrm{rac}-\mathbf{2 3}$. Acetone. 


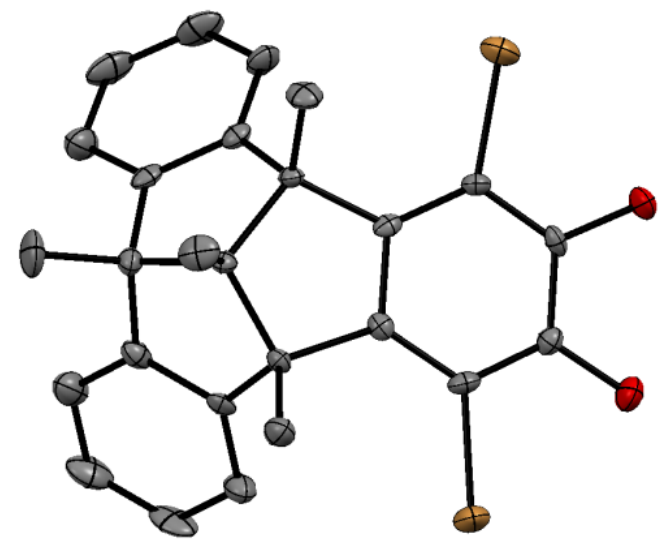

Figure S5. Crystal structure of $\mathbf{1 8}$ (ORTEP diagram, ellipsoids are shown at 30\% probability level; hydrogen atoms are omitted for clarity).

X-ray crystal data for 18 from EtOAc: $\mathrm{C}_{26} \mathrm{H}_{22} \mathrm{Br}_{2} \mathrm{O}_{2} ; M=526.25$; monoclinic; $a=$ 17.7101(9) $\AA, b=6.3887(5) \AA, c=37.130(3) \AA ; \alpha=90.00^{\circ}, \beta=95.118(7)^{\circ}, \gamma=$ $90.00^{\circ} ; V=4184.3(5) \AA^{3} ;$ space group $C 2 / \mathrm{c} ; Z=8 ; \rho_{\text {calcd }}=1.671 \mathrm{Mg} \mathrm{m}^{-3} ; T=$ $273.77(10) \mathrm{K} ; \lambda(\mathrm{Mo} \mathrm{K} \alpha)=0.71073 \AA ; 4109$ reflections collected; 2471 independent reflections; $R_{\text {int }}=0.0562 ; \mathrm{R} 1=0.0704, \mathrm{wR} 2=0.1299[\mathrm{I} \geq 2 \sigma(\mathrm{I})] . \mathrm{CCDC}-1444516$ contains the supplementary crystallographic data for $\mathbf{1 8}$.

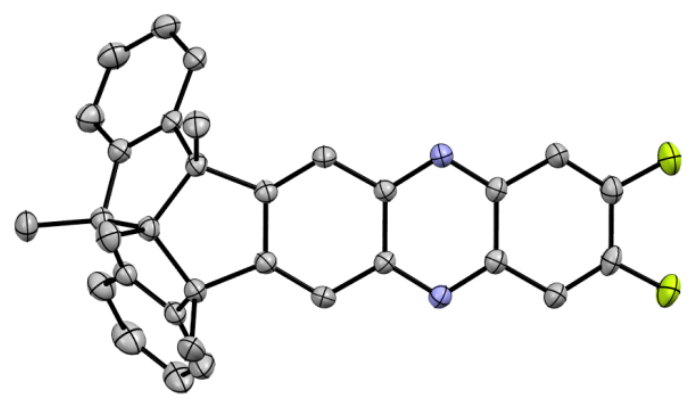

Figure S6. Crystal structure of 27b (ORTEP diagram, ellipsoids are shown at 30\% probability level; hydrogen atoms are omitted for clarity).

X-ray crystal data for $27 \mathbf{b}$ from acetone: $\mathrm{C}_{32} \mathrm{H}_{24} \mathrm{~F}_{2} \mathrm{~N}_{2} ; M=474.53$; monoclinic; $a=$ 9.6832(6) $\AA, b=17.7216(10) \AA, c=13.0766(6) \AA ; \alpha=90.00^{\circ}, \beta=90.552(5)^{\circ}, \gamma=$ $90.00^{\circ} ; V=2375.0(2) \AA^{3}$; space group $P 21 / \mathrm{c} ; Z=4 ; \rho_{\text {calcd }}=1.327 \mathrm{Mg} \mathrm{m}^{-3} ; T=293(2)$ $\mathrm{K} ; \lambda(\mathrm{Cu} \mathrm{K} \alpha)=1.54184 \AA ; 4550$ reflections collected; 3692 independent reflections; $R_{\text {int }}=0.0386 ; \mathrm{R} 1=0.0498, \mathrm{wR} 2=0.1276[\mathrm{I} \geq 2 \sigma(\mathrm{I})]$. CCDC-1028566 contains the supplementary crystallographic data for $\mathbf{2 7} \mathbf{b}$. 


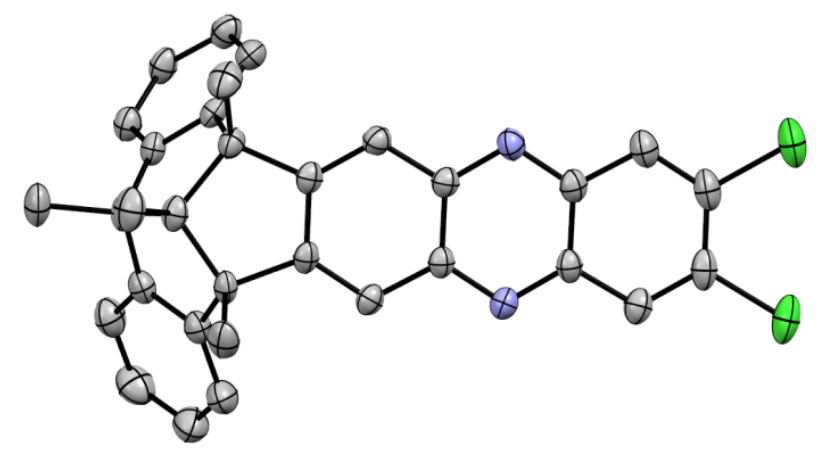

Figure S7. Crystal structure of 27c (ORTEP diagram, ellipsoids are shown at 30\% probability level; hydrogen atoms are omitted for clarity).

X-ray crystal data for $27 \mathrm{c}$ from chloroform: $\mathrm{C}_{32} \mathrm{H}_{24} \mathrm{Cl}_{2} \mathrm{~N}_{2} \cdot \mathrm{CHCl}_{3} ; M=192.86$; triclinic; $a=10.5292(8) \AA, b=11.7378(10) \AA, c=25.986(2) \AA ; \alpha=81.345(7)^{\circ}, \beta=$ $85.620(6)^{\circ}, \gamma=68.070(7)^{\circ} ; V=2944.6(4) \AA^{3}$; space group $P-1 ; Z=13 ; \rho_{\text {calcd }}=1.414$ $\mathrm{Mg} \mathrm{m}^{-3} ; T=294.81(10) \mathrm{K} ; \lambda(\mathrm{Mo} \mathrm{K} \alpha)=0.71073 \AA$; 11565 reflections collected; 6309 independent reflections; $R_{\text {int }}=0.0495 ; \mathrm{R} 1=0.0926$, wR2 $=0.2409[\mathrm{I} \geq 2 \sigma(\mathrm{I})]$. CCDC-1028567 contains the supplementary crystallographic data for $\mathbf{2 7 c}$.

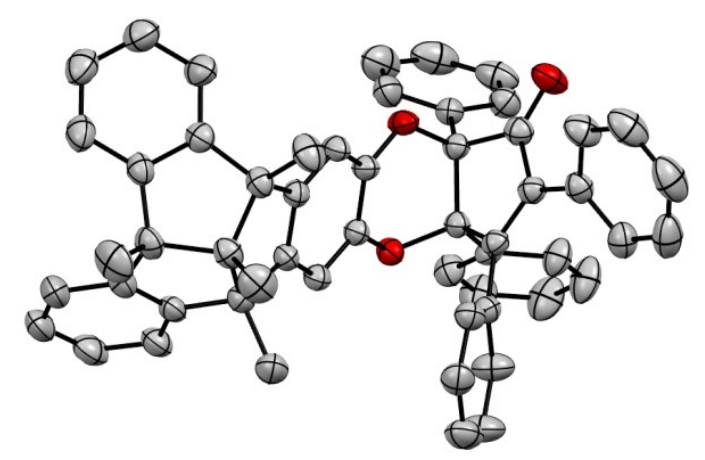

Figure S8. Crystal structure of 32 (endo-anti-stereoisomer) (ORTEP diagram, ellipsoids are shown at 30\% probability level; hydrogen atoms are omitted for clarity).

X-ray crystal data for 32 (endo-anti-stereoisomer) from chloroform: $\mathrm{C}_{55} \mathrm{H}_{42} \mathrm{O}_{3} ; M=$ 750.88; monoclinic; $a=10.8938(19) \AA, b=33.139(5) \AA, c=11.704(2) \AA ; \alpha=90^{\circ}, \beta$ $=103.521(17)^{\circ}, \gamma=90^{\circ} ; V=4108.3(12) \AA^{3}$; space group $P 21 / \mathrm{n} ; Z=4 ; \rho_{\text {calcd }}=1.214$ $\mathrm{Mg} \mathrm{m}^{-3} ; T=293(2) \mathrm{K} ; \lambda($ Mo K $\alpha)=0.7107 \AA \AA ; 085$ reflections collected; 3988 independent reflections; $R_{\text {int }}=0.0570 ; \mathrm{R} 1=0.0792$, wR2 $=0.1812[\mathrm{I} \geq 2 \sigma(\mathrm{I})]$. CCDC-1028572 contains the supplementary crystallographic data for $\mathbf{3 2}$ (endo-anti-stereoisomer). 
(3) Crystal packing of catechols $\mathbf{1 7}$ and $\mathbf{2 3}$ in different solvents.

Good-quality crystals of the two catechols $\mathbf{1 7}$ and $\mathbf{2 3}$ were obtained from a number of solvents, such as ethyl acetate/petroleum ether, acetone and THF. The X-ray structure analyses performed with these various single crystals were revealing (Figure S8). In all cases, the crystals contained trapped solvent molecules in a 1:1 ratio. In the crystal of 17 obtained from ethyl acetate/petroleum ether, all of the TBTQ bowls adopt the same orientation within each single layer, thus forming 1-D molecular chains. These chains are connected by hydrogen bonds between the hydroxyl groups of two adjacent TBTQ molecules. In addition, hydrogen bonds exist between the carbonyl group of each ethyl acetate guest and one of the hydroxyl groups of host 17 . The crystals of $\mathbf{1 7}$ obtained from THF consist of dimers that are held together in a zig-zag-syn coordination by two parallel $\mathrm{O}-\mathrm{H} \cdots \mathrm{O}$ hydrogen bonds. In this case, the solvent molecule is coordinated to two diametric groups of the eight-membered rings thus formed. The molecules of the $C_{1}$-symmetrical isomer $( \pm)$-23 obtained from acetone are packed in a similar way, again zig-zag-syn, and each dimer consists of a pair of the $(P)$ - or $(M)$-enantiomers.
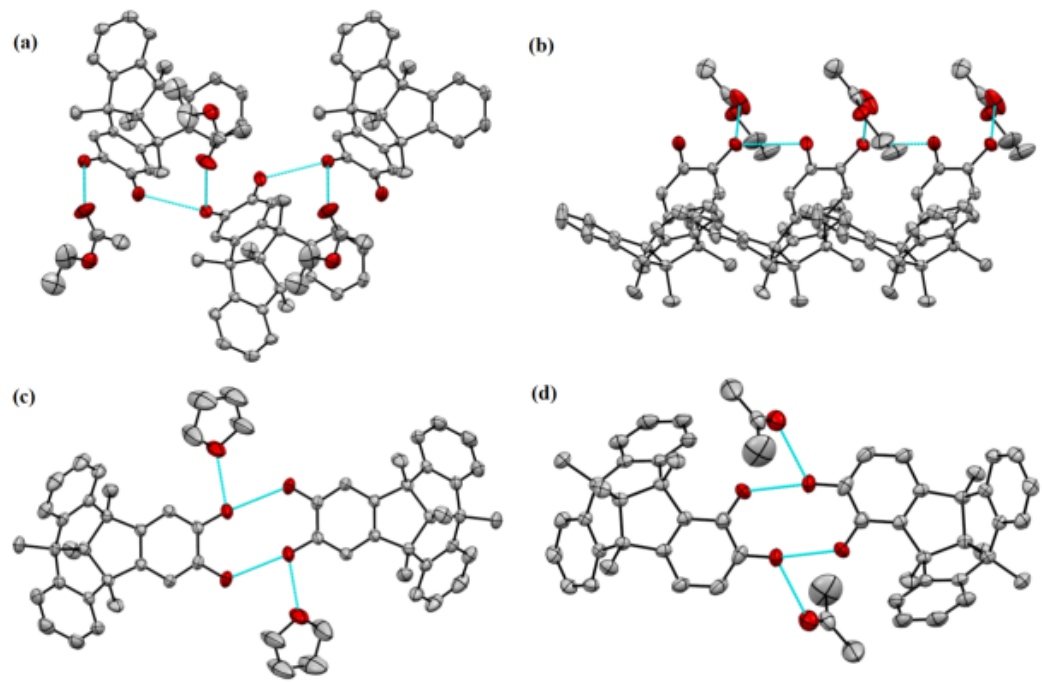

Figure S9. Crystal structure (ORTEP diagrams) of TBTQ-catechol 17 as obtained from EtOAc/petroleum ether (a) viewed along the crystallographic $a$ axis and (b) along the $b$ axis; (c) catechol $\mathbf{1 7}$ as obtained from THF, viewed along the $b$ axis; and (d) rac-catechol 23, as obtained from acetone, viewed along the $c$ axis. Ellipsoids are shown at $30 \%$ probability level; hydrogen atoms are omitted for clarity. 
(4) Crystal packing of phenazines $27 \mathbf{b}$ and $27 \mathbf{c}$.

The crystal structure of the difluorophenazine $\mathbf{2 7} \mathbf{b}$ shows that the TBTQ moieties are stacked parallel to the polar $c$ axis and that the two neighboring phenazine moieties are almost parallel to each other at distances of 3.30-3.47 $\AA$ (Figure 3a). Moreover, pairs of $\mathrm{C}-\mathrm{F} \cdots \mathrm{H}-\mathrm{C}^{\text {methyl }}$ hydrogen bonds add to the interactions between two adjacent molecules with distances of $2.640 \AA$. The fluorinated parts of a pair of adjacent molecules are mutually stacked on top of the inner benzene rings of the other molecule, pointing to significant intermolecular $\pi-\pi$ interactions. This structural motif is reminiscent to the strong interaction between the co-crystallized hexafluorobenzene and benzene rings. ${ }^{1}$ In the case of the dichloro derivative $27 \mathbf{c}$, the molecules are stacked in a very different way (Figure 3b). Compound 27c was found to form dimers with coordinated pyrazine units but the incorporated molecules of chloroform were coordinated by $\mathrm{Cl} \cdots \mathrm{N}$ bonds with bond lengths of $3.138 \AA$. The neighboring molecules also showed $\mathrm{CH}^{\cdots} \pi \pi$ interactions (with distances of $2.863 \AA$ ) as another driving force that controlled the assembly of $27 \mathbf{c}$ in the solid state.
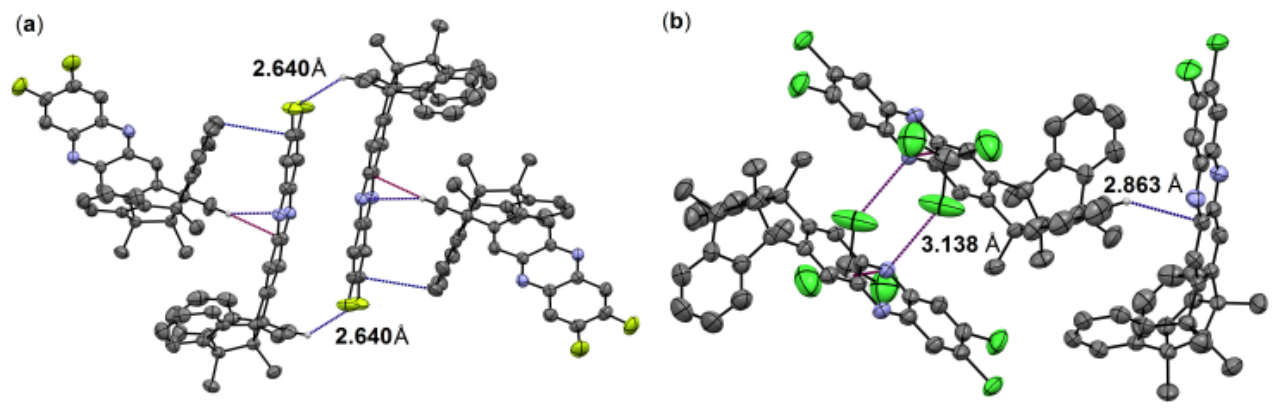

Figure S10. Crystal structures of TBTQ-phenazines in the solid state (ORTEP diagrams). (a) Phenazine 27b, as obtained from EtOAc/petroleum ether, viewed along the crystallographic $b$ axis; (b) phenazine 27c, as obtained from $\mathrm{CDCl}_{3}$, viewed along the $b$ axis. Ellipsoids are shown at $30 \%$ probability level.

(1) Lindner, B. D.; Engelhart, J. U.; Märken, M.; Tverskoy, O.; Appleton, A. L.; Rominger, F.; Hardcastle, K. I.; Enders, M.; Bunz, U. H. F. Chem. - Eur. J. 2012, $18,4627-4633$. 